\title{
Large deviations conditioned on large deviations II: Fluctuating hydrodynamics
}

\author{
Bernard Derrida . Tridib Sadhu
}

the date of receipt and acceptance should be inserted later

\begin{abstract}
For diffusive many-particle systems such as the SSEP (symmetric simple exclusion process) or independent particles coupled with reservoirs at the boundaries, we analyze the density fluctuations conditioned on current integrated over a large time. We determine the conditioned large deviation function of density by a microscopic calculation. We then show that it can be expressed in terms of the solutions of Hamilton-Jacobi equations, which can be written for general diffusive systems using a fluctuating hydrodynamics description.
\end{abstract}

Keywords Large deviation function, fluctuating hydrodynamics, non-equilibrium steady state, symmetric simple exclusion process.

PACS 05.40.-a, 05.70.Ln, 05.10.Gg

\section{Introduction}

In recent years, there has been a growing interest [1 9] in characterizing trajectories conditioned on rare events. Such questions appear in a wide range of topics, including protein folding [10], chemical reactions [11, 12, stochastic models of gene expression [13], atmospheric activities [14], glassy systems [15, 16], disordered media [17, etc.. One motivation is to find efficient algorithms where the rare events become typical such that they are computationally easy to generate $2,11,18,20$. To calculate the probability of rare events one needs to understand how these rare events are created and how they relax. In these activities, a major interest concerns conditioning on an atypical value of an empirical observable, such as the integrated current, the empirical density, the entropy production, and the activity 21 27]. How do the fluctuations get affected by such conditioning, and what is the

Bernard Derrida

Collège de France, 11 place Marcelin Berthelot, 75231 Paris Cedex 05 - France.

bernard.derrida@college-de-france.fr

Tridib Sadhu

Tata Institute of Fundamental Research, Homi Bhabha Road, Mumbai 400005.

Collège de France, 11 place Marcelin Berthelot, 75231 Paris Cedex 05 - France.

tridib@theory.tifr.res.in 


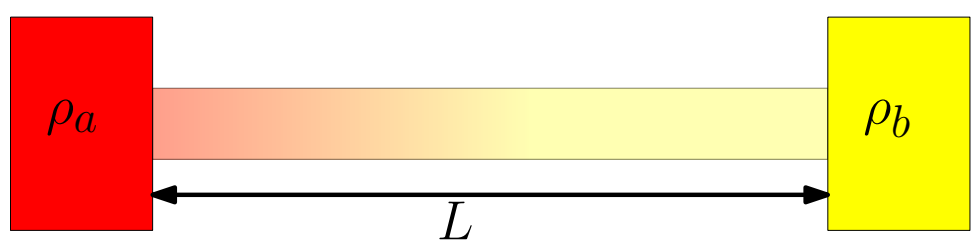

Fig. 1 A diffusive system of length $L$ coupled with two reservoirs at different densities $\rho_{a}$ and $\rho_{b}$ at the boundary.

effective dynamics in this conditioned ensemble? These are questions we address in this present work.

For Markov processes, in particular, for systems with few degrees of freedom and evolving according to a Langevin equation, it is known [1, 8, 15, 16, 22, 28, 32] how to describe the conditioned dynamics when the time window for the empirical observable is large. In this paper, we see how the same ideas 28] can be extended to systems with many degrees of freedom.

We work here in the steady state of a one-dimensional diffusive system of length $L$ coupled with reservoirs at the boundary, as shown in Figure 1. Under a diffusive re-scaling of space $i$ and time $\tau$, defining $\left(\frac{i}{L}, \frac{\tau}{L^{2}}\right) \equiv(x, t)$ for large $L$, these systems are described [33 38] by a hydrodynamic density $\rho(x, t)$ and current $j(x, t)$ whose time evolution is governed by a fluctuating hydrodynamics equation

$$
\partial_{t} \rho(x, t)=-\partial_{x} j(x, t) \quad \text { with } \quad j(x, t)=-D(\rho(x, t)) \partial_{x} \rho(x, t)+\eta(x, t)
$$

where $D(\rho)$ is the diffusivity and $\eta(x, t)$ is a Gaussian white noise with zero mean and covariance

$$
\left\langle\eta(x, t) \eta\left(x^{\prime}, t^{\prime}\right)\right\rangle=\frac{1}{L} \sigma(\rho(x, t)) \delta\left(x-x^{\prime}\right) \delta\left(t-t^{\prime}\right)
$$

where $\sigma(\rho)$ is the mobility. The density $\rho(x, t)$ could be the density of particles or of energy depending on the system. The microscopic details of the system are embedded in the functions $D(\rho)$ and $\sigma(\rho)$. The pre-factor $\frac{1}{L}$ in the covariance of $\eta(x, t)$ is due to coarsegraining, which makes the noise strength weak for large $L$ 33, 35, 38, 39.

Our interest is in the statistics of the steady state density $\rho(x)$ when conditioned on the integrated current $Q_{T}$ over a time interval $[0, T]$, for large $T$. Individual statistics of $\rho(x)$ and $Q_{T}$ have already been studied 35, 39, 48. For the conserved dynamics (1), when the density is bounded inside the bulk, the probability of $Q_{T}$ for large $T$ is independent of where the current is measured, and $P\left(Q_{T}\right)$ has a large deviation description [41, 42, 48]. On the other hand, in the steady state, the probability of $\rho(x)$ has also a large deviation form when the system size $L$ is large [35, 39, 40, 43. These two probabilities are given by

$$
P\left(Q_{T}=q T\right) \sim e^{-T L \Phi(q)} \quad \text { and } \quad P[\rho(x)] \sim e^{-L \mathcal{F}[\rho(x)]}
$$

for $T$ and $L$ both being large, where $\Phi(q)$ and $\mathcal{F}[\rho(x)]$ are the corresponding large deviation functions. Here, the precise meaning of the symbol $\sim$ is that $\lim _{L \rightarrow \infty} \lim _{T \rightarrow \infty} \frac{\log P\left(Q_{T}=q T\right)}{L T}=-\Phi(q)$ and $\lim _{L \rightarrow \infty} \frac{\log P[\rho(x)]}{L}=-\mathcal{F}[\rho(x)]$. 


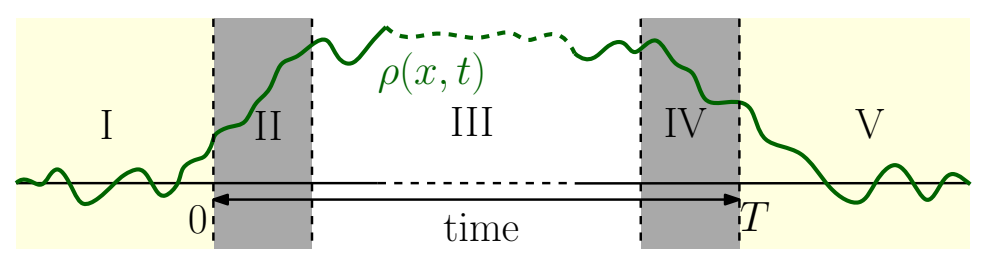

Fig. 2 A schematic time evolution of the density profile $\rho(x, t)$ when conditioned on $Q_{T}^{(\alpha)}$ in (3) measured over a large time interval $[0, T]$. The system starts in its steady state far in the past, then reaches a quasi-stationary state for $1 \ll t$ and $1 \ll T-t$, and finally for $t \gg T$ it relaxes to its steady state. Different shaded regions denote different parts of the evolution: (I) $t<0$, (II) $t \geq 0$ but small, (III) $1 \ll t$ and $1 \ll T-t$, (IV) $T-t>0$ but small, and (V) $t \geq T$.

Their joint statistics, and equivalently, the conditioned probability of $\rho(x)$ for a given value of $Q_{T}$ depends on where the latter is measured. Taking this into account, we define an empirical observable

$$
Q_{T}^{(\alpha)}=\int_{0}^{1} d x \alpha(x) \int_{0}^{T} d t j(x, t) \quad \text { with } \quad \int_{0}^{1} d x \alpha(x)=1 .
$$

Here, $\alpha(x)$ is arbitrary except for the normalization ${ }^{1}$ in $[3]$.

We denote by $\mathcal{P}_{t}^{(\alpha)}[\rho(x) \mid Q]$ the conditioned probability of a density profile $\rho(x)$ at time $t$ given that $Q_{T}^{(\alpha)}$ takes value $Q$ while the system is in its steady state. For large $T$ and $L$, it has the large deviation form

$$
\mathcal{P}_{t}^{(\alpha)}[\rho(x) \mid Q=q T] \sim e^{-L \psi_{t}^{(\alpha)}[\rho(x), q]}
$$

where $\psi_{t}^{(\alpha)}[\rho(x), q]$ is the conditioned large deviation function.

Our goal is to determine this large deviation function $\psi_{t}^{(\alpha)}[\rho(x), q]$. In this paper, we will give this function at $t=0$, in the intermediate quasi-stationary state $(t \gg 1$ and $T-t \gg 1$ ), and at $t=T$ (indicated in Figure 2) for diffusing independent particles and for the symmetric simple exclusion process [35]. Our results for the latter are in a perturbation expansion in small density. A hydrodynamic description of these two examples is given by (1) with $D(\rho)=1$ and $\sigma(\rho)=2 \rho$ for the diffusing independent particles, and $D(\rho)=1$ and $\sigma(\rho)=2 \rho(1-\rho)$ for the symmetric simple exclusion process.

We shall start by a microscopic calculation on these models where we determine the conditioned probability in terms of the left and right eigenvectors associated to the largest eigenvalue of a tilted matrix [28. Then, taking a hydrodynamic limit of the conditioned probability we derive $\psi_{t}^{(\alpha)}[\rho(x), q]$. For example, in the quasi-stationary state (regime III in Figure 2), we will see that

$$
\psi_{t}^{(\alpha)}[\rho(x), q] \equiv \psi_{\mathrm{qs}}[\rho(x), q]=\psi_{\text {left }}^{(\alpha)}[\rho(x), q]+\psi_{\text {right }}^{(\alpha)}[\rho(x), q]
$$

up to an additive constant ${ }^{2}$ (subscript "qs" denotes "quasi-stationary"), where $\psi_{\text {left }}^{(\alpha)}$ and $\psi_{\text {right }}^{(\alpha)}$ are related [28] to the left and right eigenvectors of a tilted matrix

1 The normalization for $\alpha(x)$ in 3 ensures that the probability $P\left(Q_{T}^{(\alpha)}=q T\right)$ has the large deviation form (2) with the same large deviation function $\Phi(q)$, independent of $\alpha(x)$.

2 We shall show that the $\alpha(x)$ dependence cancels in the expression 5 for $\psi_{\mathrm{qs}}[\rho(x), q]$. In other regions of Figure 2 e.g. at $t=0$ and $t=T, \psi_{t}^{(\alpha)}[\rho(x), q]$ depends on $\alpha(x)$. 


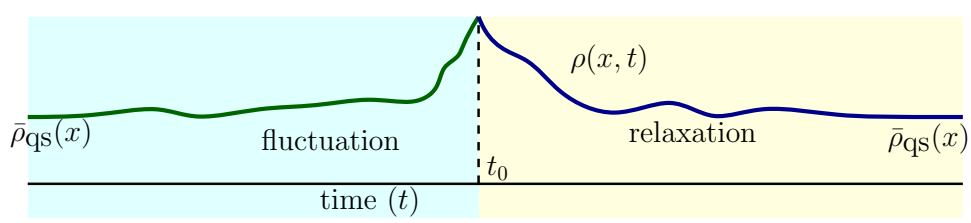

Fig. 3 A schematic path $\rho(x, t)$ leading to a fluctuation in density at $t=t_{0}$ in the quasistationary regime, and its subsequent relaxation to the quasi-stationary density $\bar{\rho}_{\mathrm{qs}}(x)$.

(see Section 2.5 for a precise definition). We will see later that these two functions $\psi_{\text {left }}^{(\alpha)}[\rho(x), q]$ and $\psi_{\text {right }}^{(\alpha)}[\rho(x), q]$ have in fact the following physical interpretation in terms of the large deviation function defined in (4).

$$
\begin{aligned}
\psi_{\text {left }}^{(\alpha)}[\rho(x), q] & =\psi_{0}^{(\alpha)}[\rho(x), q]-\mathcal{F}[\rho(x)] \\
\psi_{\text {right }}^{(\alpha)}[\rho(x), q] & =\psi_{T}^{(\alpha)}[\rho(x), q]
\end{aligned}
$$

up to an additive constant, where $\mathcal{F}[\rho(x)]$ is the unconditioned large deviation function of the density, as defined in (2).

In addition, we shall see that the conditioned dynamics for large $T$, can be effectively described by a fluctuating hydrodynamics equation with an additional driving field, which can be expressed in terms of $\psi_{\text {left }}^{(\alpha)}$ and $\psi_{\text {right }}^{(\alpha)}$. For example, in the quasi-stationary regime, the path $\rho(x, t)$ leading to a fluctuation at $t_{0}$ with $1 \ll t_{0}$ and $1 \ll T-t_{0}$ (illustrated in Figure 3 ), follows, for $t<t_{0}$,

$$
\partial_{t} \rho(x, t)=\partial_{x}\left\{D(\rho) \partial_{x} \rho-\sigma(\rho)\left(\Phi^{\prime}(q) \alpha(x)+\partial_{x} \frac{\delta \psi_{\text {right }}^{(\alpha)}}{\delta \rho(x, t)}\right)+\eta(x, t)\right\}
$$

with $\eta(x, t)$ being a Gaussian white noise of zero mean and covariance (1b). Similarly, the path $\rho(x, t)$ for subsequent relaxation $\left(t \geq t_{0}\right)$ follows

$$
\partial_{t} \rho(x, t)=\partial_{x}\left\{D(\rho) \partial_{x} \rho-\sigma(\rho)\left(\Phi^{\prime}(q) \alpha(x)-\partial_{x} \frac{\delta \psi_{\text {left }}^{(\alpha)}}{\delta \rho(x, t)}\right)+\eta(x, t)\right\}
$$

In the later part of this paper, we shall show that these results are consistent with a macroscopic approach starting from (1) for a rather general $D(\rho)$ and $\sigma(\rho)$. In this approach, $\psi_{\text {left }}^{(\alpha)}$ and $\psi_{\text {right }}^{(\alpha)}$ are solution of the Hamilton-Jacobi equations

$$
\begin{aligned}
& \int_{0}^{1} d x\left\{\frac{\sigma(\rho)}{2}\left(\partial_{x} \frac{\delta \psi_{\text {left }}^{(\alpha)}}{\delta \rho(x, t)}-\Phi^{\prime}(q) \alpha(x)+\frac{D(\rho) \partial_{x} \rho}{\sigma(\rho)}\right)^{2}-\frac{\left(D(\rho) \partial_{x} \rho\right)^{2}}{2 \sigma(\rho)}\right\} \\
& =\Phi^{\prime}(q) q-\Phi(q) \\
& \int_{0}^{1} d x\left\{\frac{\sigma(\rho)}{2}\left(\partial_{x} \frac{\delta \psi_{\text {right }}^{(\alpha)}}{\delta \rho(x, t)}+\Phi^{\prime}(q) \alpha(x)-\frac{D(\rho) \partial_{x} \rho}{\sigma(\rho)}\right)^{2}-\frac{\left(D(\rho) \partial_{x} \rho\right)^{2}}{2 \sigma(\rho)}\right\} \\
& =\Phi^{\prime}(q) q-\Phi(q)
\end{aligned}
$$

One should note that all our results for the conditioned process are in the large $T$ limit. 


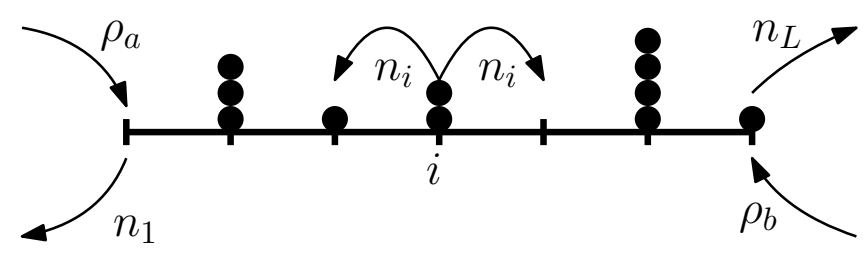

Fig. 4 Transition rates for independent particles on a one-dimensional chain coupled with reservoirs of density $\rho_{a}$ and $\rho_{b}$. The number of particles $n_{i} \geq 0$ at a site $i$ is arbitrary.

We present this paper in the following order. In Section 2, we discuss the microscopic framework for analyzing the conditioned probability in a general lattice gas model and introduce conditioned large deviation function in the hydrodynamic limit. Our calculation is in a weighted ensemble, which is known [1, 8, 22, 28, 30. to be equivalent to the conditioned process in the large $T$ limit (through an equivalence of ensembles). We use this procedure to derive $\psi_{t}^{(\alpha)}[\rho(x), q]$ for the diffusing independent particles in Section 3 , and for the symmetric simple exclusion process in Section 4. Using this microscopic approach we describe the conditioned dynamics in Section 5. In Section 6 and Section 7, we show how our expressions of the large deviation function $\psi_{t}^{(\alpha)}[\rho(x), q]$ fit with the Hamilton-Jacobi equations derived from a macroscopic approach starting from the fluctuating hydrodynamics description (1).

\section{Microscopic analysis using the tilted matrix}

Let us first recall a few earlier results [1, 8, 16, 22, 28, 31, 49] in a Markov process conditioned on an empirical measure by writing them for the two examples: (a) diffusing independent particles and (b) the symmetric simple exclusion process. These are defined on a finite one-dimensional lattice of $L$ sites where particles jump between neighboring sites following a continuous time $\tau$ update rule (see Figure 4 and Figure 5). The jump rates at the boundary correspond to coupling to reservoirs of density $\rho_{a}$ and $\rho_{b}$ 35. In both examples, a configuration is specified by the set of occupation variables $\mathbf{n} \equiv\left\{n_{1}, \ldots, n_{L}\right\}$.

The microscopic analogue of the empirical observable $(3)$ is

$$
\mathcal{Q}_{N}^{(\boldsymbol{\lambda})}=\sum_{i=0}^{L} \lambda_{i} \times \begin{gathered}
\text { number of jumps from site } i \text { to } i+1 \\
\text { during the time interval }[0, N]
\end{gathered}
$$

where $\boldsymbol{\lambda} \equiv\left\{\lambda_{0}, \ldots, \lambda_{L}\right\}$ with $\lambda_{i}$ being real valued parameters. A jump from site $i+1$ to $i$ is counted as a jump from site $i$ to $i+1$ with a negative sign. (Here, $i=0$ denotes the left reservoir and $i=L+1$ denotes the right reservoir.) Similar to the condition in (3) we consider

$$
\sum_{i=0}^{L} \lambda_{i}=1
$$




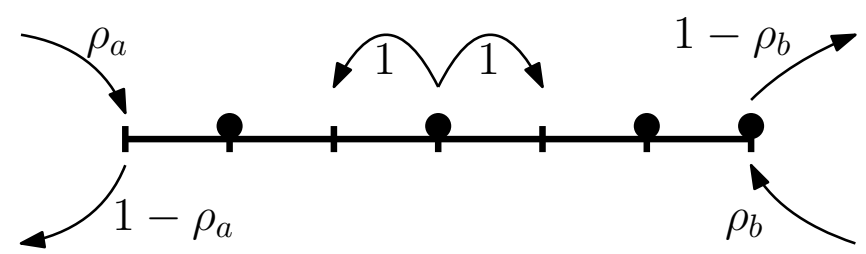

Fig. 5 Transition rates of particles in the symmetric simple exclusion process on a onedimensional chain coupled with reservoirs of density $\rho_{a}$ and $\rho_{b}$. A site is occupied at most by one particle at a time.

\subsection{Tilted matrix}

Instead of conditioning on the value of $\mathcal{Q}_{N}^{(\boldsymbol{\lambda})}$, it is more convenient [1, 8, 28, 30, 49 . to work in the ensemble where events are weighted by a factor $e^{\kappa \mathcal{Q}_{N}^{(\boldsymbol{\lambda})}}$. By analogy [1, 8, 22, 28, 30, 32, 50, with equilibrium thermodynamics, we shall refer to this ensemble as the canonical ensemble and the ensemble where $\mathcal{Q}_{N}^{(\boldsymbol{\lambda})}$ is fixed as the micro-canonical ensemble. For large $N$, these two ensembles are equivalent (see Section 2.3.

In the canonical ensemble, we need to introduce the following tilted matrix 28 .

$$
\mathcal{M}_{\kappa}^{(\boldsymbol{\lambda})}\left(\mathbf{n}^{\prime}, \mathbf{n}\right)= \begin{cases}e^{\kappa \sum_{i=0}^{L} \lambda_{i} \mathcal{J}_{i}\left(\mathbf{n}^{\prime}, \mathbf{n}\right)} \mathcal{M}_{0}\left(\mathbf{n}^{\prime}, \mathbf{n}\right) & \text { for } \mathbf{n}^{\prime} \neq \mathbf{n} \\ -\sum_{\mathbf{n}^{\prime \prime} \neq \mathbf{n}} \mathcal{M}_{0}\left(\mathbf{n}^{\prime \prime}, \mathbf{n}\right) & \text { for } \mathbf{n}^{\prime}=\mathbf{n}\end{cases}
$$

where $\mathcal{M}_{0}\left(\mathbf{n}^{\prime}, \mathbf{n}\right)$ is the transition rate from configuration $\mathbf{n}$ to $\mathbf{n}^{\prime}$ in the original (without condition) dynamics and for this transition,

$$
\mathcal{J}_{i}\left(\mathbf{n}^{\prime}, \mathbf{n}\right)= \begin{cases}1, & \text { if a particle jumps from } i \text { to } i+1, \\ -1, & \text { if a particle jumps from } i+1 \text { to } i, \\ 0, & \text { if no particle jumps between } i \text { and } i+1 .\end{cases}
$$

The scaled cumulant generating function of $\mathcal{Q}_{N}^{(\boldsymbol{\lambda})}$, defined by

$$
\mu(\kappa)=\lim _{N \rightarrow \infty} \frac{\log \left\langle e^{\kappa \mathcal{Q}_{N}^{(\boldsymbol{\lambda})}}\right\rangle}{N},
$$

is the largest eigenvalue of $\mathcal{M}_{\kappa}^{(\boldsymbol{\lambda})}[28]$ such that

$$
\begin{aligned}
& \sum_{\mathbf{n}} \mathcal{M}_{\kappa}^{(\boldsymbol{\lambda})}\left(\mathbf{n}^{\prime}, \mathbf{n}\right) \mathcal{R}^{(\kappa, \boldsymbol{\lambda})}(\mathbf{n})=\mu(\kappa) \mathcal{R}^{(\kappa, \boldsymbol{\lambda})}\left(\mathbf{n}^{\prime}\right) \\
& \sum_{\mathbf{n}^{\prime}} \mathcal{L}^{(\kappa, \boldsymbol{\lambda})}\left(\mathbf{n}^{\prime}\right) \mathcal{M}_{\kappa}^{(\boldsymbol{\lambda})}\left(\mathbf{n}^{\prime}, \mathbf{n}\right)=\mu(\kappa) \mathcal{L}^{(\kappa, \boldsymbol{\lambda})}(\mathbf{n})
\end{aligned}
$$

where $\mathcal{R}^{(\kappa, \boldsymbol{\lambda})}$ and $\mathcal{L}^{(\kappa, \boldsymbol{\lambda})}$ are the associated right and left eigenvectors, respectively.

At times of our interest, namely $\tau=0, \tau=N$, and in the quasi-stationary regime, the probability $P_{\tau}^{(\kappa, \boldsymbol{\lambda})}(\mathbf{n})$ of a configuration $\mathbf{n}$ in the canonical ensemble can be expressed (see [8, 28, 51]) in terms of these eigenvectors up to normalization constants. 
- at time $\tau=0$,

$$
P_{0}^{(\kappa, \boldsymbol{\lambda})}(\mathbf{n})=\mathcal{L}^{(\kappa, \boldsymbol{\lambda})}(\mathbf{n}) \mathcal{R}^{(0, \boldsymbol{\lambda})}(\mathbf{n})
$$

- at time $\tau=N$,

$$
P_{N}^{(\kappa, \boldsymbol{\lambda})}(\mathbf{n})=\mathcal{R}^{(\kappa, \boldsymbol{\lambda})}(\mathbf{n})
$$

- and in the quasi-stationary regime, i.e. $1 \ll \tau$ with $1 \ll N-\tau$,

$$
P_{\mathrm{qs}}^{(\kappa, \boldsymbol{\lambda})}(\mathbf{n})=\mathcal{L}^{(\kappa, \boldsymbol{\lambda})}(\mathbf{n}) \mathcal{R}^{(\kappa, \boldsymbol{\lambda})}(\mathbf{n})
$$

\subsection{Dependence on $\lambda$}

In our examples, the number of particles is conserved inside the bulk of the system, which means

$$
\mathcal{J}_{i-1}\left(\mathbf{n}^{\prime}, \mathbf{n}\right)-\mathcal{J}_{i}\left(\mathbf{n}^{\prime}, \mathbf{n}\right)=n_{i}^{\prime}-n_{i}
$$

for all $1 \leq i \leq L$ and equivalently

$$
\mathcal{J}_{i}\left(\mathbf{n}^{\prime}, \mathbf{n}\right)=\mathcal{J}_{0}\left(\mathbf{n}^{\prime}, \mathbf{n}\right)-\sum_{j=1}^{i}\left(n_{j}^{\prime}-n_{j}\right)
$$

This conservation of particles leads to a symmetry of the tilted matrix and its eigenvectors. To see this, we use the above relation in 11 . Here, using $\sum_{i=1}^{L} \lambda_{i} \sum_{j=1}^{i} n_{j}=$ $\sum_{i=1}^{L} n_{i} \sum_{j \geq i}^{L} \lambda_{j}$ and the normalization 10 we get

$$
\left[e^{\kappa \sum_{i=1}^{L} n_{i}^{\prime} \sum_{j \geq i}^{L} \lambda_{j}}\right] \mathcal{M}_{\kappa}^{(\boldsymbol{\lambda})}\left(\mathbf{n}^{\prime}, \mathbf{n}\right)\left[e^{-\kappa \sum_{i=1}^{L} n_{i} \sum_{j \geq i}^{L} \lambda_{j}}\right]=\mathcal{M}_{\kappa}\left(\mathbf{n}^{\prime}, \mathbf{n}\right)
$$

where $\mathcal{M}_{\kappa}$ is the tilted matrix for the case where $\lambda_{0}=1$ and $\lambda_{i}=0$ for rest of the sites. Denoting the eigenvectors of $\mathcal{M}_{\kappa}$ as $\left(\mathcal{R}^{(\kappa)}, \mathcal{L}^{(\kappa)}\right)$, we get

$$
\begin{aligned}
\mathcal{R}^{(\kappa, \boldsymbol{\lambda})}(\mathbf{n})\left[e^{\kappa \sum_{i=1}^{L} n_{i} \sum_{j \geq i}^{L} \lambda_{j}}\right] & =\mathcal{R}^{(\kappa)}(\mathbf{n}) \\
\mathcal{L}^{(\kappa, \boldsymbol{\lambda})}(\mathbf{n})\left[e^{-\kappa \sum_{i=1}^{L} n_{i} \sum_{j \geq i}^{L} \lambda_{j}}\right] & =\mathcal{L}^{(\kappa)}(\mathbf{n}) .
\end{aligned}
$$

Moreover, the eigenvalue $\mu(\kappa)$ is same in the two cases, which shows that it is independent of $\lambda$.

This gives the $\lambda$-dependence of the probabilities (14). For example, using (15) in $14 \mathrm{c}$ we see that in the quasi-stationary regime, the probability $P_{\mathrm{qs}}^{(\kappa, \boldsymbol{\lambda})}$ is independent $\boldsymbol{\lambda}$ (given the normalization in 100 ),

$$
P_{\mathrm{qS}}^{(\kappa, \boldsymbol{\lambda})}(\mathbf{n}) \equiv P_{\mathrm{qS}}^{(\kappa)}(\mathbf{n})
$$


2.3 An equivalence of ensembles: canonical vs. micro-canonical

For large time $N$, there is [1, 8, 22, 28, 30, an equivalence between the canonical ensemble and the micro-canonical ensemble, where $\mathcal{Q}_{N}^{(\boldsymbol{\lambda})}$ is fixed. In this equivalence, the conditioned probability $\mathcal{P}_{\tau}^{(\boldsymbol{\lambda})}(\mathbf{n} \mid \mathcal{Q})$ of a configuration $\mathbf{n}$ at time $\tau$ given $\mathcal{Q}_{N}^{(\boldsymbol{\lambda})}=\mathcal{Q}$ is related to the probability $P_{\tau}^{(\kappa, \boldsymbol{\lambda})}(\mathbf{n})$ in the canonical ensemble by

$$
\mathcal{P}_{\tau}^{(\boldsymbol{\lambda})}(\mathbf{n} \mid \mathcal{Q}=v N) \simeq P_{\tau}^{\left(\phi^{\prime}(v), \boldsymbol{\lambda}\right)}(\mathbf{n}) \quad \text { for large time } N,
$$

where $\phi(v)$ is the Legendre transform of the eigenvalue $\mu(\kappa)$, defined by

$$
\phi(v)=v \kappa-\mu(\kappa) \quad \text { with } \mu^{\prime}(\kappa)=v .
$$

This also means $35,41,42$, 48, that the probability of $\mathcal{Q}_{N}^{(\boldsymbol{\lambda})}$ has a large deviation form given by

$$
P\left(\mathcal{Q}_{N}^{(\boldsymbol{\lambda})}=v N\right) \sim e^{-N \phi(v)} \quad \text { for large time } N
$$

In this work, our results for the micro-canonical ensemble are obtained from the canonical ensemble using the equivalence (17).

\subsection{Hydrodynamic limit}

Our main interest is the probability in the hydrodynamic limit, which is defined in the scaled coordinates $(x, t) \equiv\left(\frac{i}{L}, \frac{\tau}{L^{2}}\right)$ for large $L$, with $N=L^{2} T$ and $\lambda_{i}=\frac{1}{L} \alpha(x)$ such that

$$
\mathcal{Q}_{N}^{(\boldsymbol{\lambda})} \simeq L Q_{T}^{(\alpha)} \quad \text { for large } L,
$$

with $Q_{T}^{(\alpha)}$ given in $(3)$. In this hydrodynamic limit,

$$
\phi\left(v=\frac{q}{L}\right) \simeq \frac{1}{L} \Phi(q), \quad \mu(\kappa) \simeq \frac{1}{L} \chi(\kappa)
$$

and they are related (due to 18 ) by

$$
\Phi(q)=q \kappa-\chi(\kappa) \quad \text { for } \chi^{\prime}(\kappa)=q .
$$

This scaling (20) is well known [4, 42, 47, 48, and this will be confirmed in our examples.

For the probability of occupation variables $\mathbf{n}$, taking a hydrodynamic limit means [35, 37, 52 coarse-graining the system over boxes of width $w$ with $1 \ll w \ll$ $L$, such that each box has a uniform density $\rho(x)$ which varies smoothly on the hydrodynamic scale $x$. If $P(\mathbf{n})$ is the probability of a microscopic configuration $\mathbf{n}$, then the probability $P[\rho(x)]$ of a hydrodynamic density profile $\rho(x)$ is

$$
\sum_{\mathbf{n} \in \rho(x)} P(\mathbf{n}) \simeq P[\rho(x)]
$$

where the summation is over all $\mathbf{n}$ that correspond to the profile $\rho(x)$ (see for example Section 3 . 
The right eigenvector $\mathcal{R}^{(\kappa, \boldsymbol{\lambda})}$ has an interpretation of a probability (see $14 \mathrm{~b}$ ) and therefore, its hydrodynamic limit is similarly defined by

$$
\sum_{\mathbf{n} \in \rho(x)} \mathcal{R}^{(\kappa, \boldsymbol{\lambda})}(\mathbf{n}) \simeq r^{(\kappa, \alpha)}[\rho(x)]
$$

In comparison, $\mathcal{L}^{(\kappa, \boldsymbol{\lambda})}$ by itself does not have an interpretation of a probability. Considering 14a, 14c), we define the hydrodynamic limit for the left eigenvector as

$$
\mathcal{L}^{(\kappa, \boldsymbol{\lambda})}(\mathbf{n}) \simeq \ell^{(\kappa, \alpha)}[\rho(x)]
$$

for each configuration $\mathbf{n}$ that corresponds to the profile $\rho(x)$. (Note that in contrast to 23a there is no summation over $\mathbf{n}$ in $23 \mathrm{~b}$.)

Using this construction of the hydrodynamic limit in 23), we see that the probability in (14) leads to the probability of density $\rho(x)$, which

- at $t=0$ is given by

$$
P_{t=0}^{(\kappa, \alpha)}[\rho(x)] \simeq \ell^{(\kappa, \alpha)}[\rho(x)] r^{(0, \alpha)}[\rho(x)]
$$

- at $t=T$ is given by

$$
P_{t=T}^{(\kappa, \alpha)}[\rho(x)] \simeq r^{(\kappa, \alpha)}[\rho(x)],
$$

- and in the quasi-stationary regime $(1 \ll t$ and $1 \ll T-t)$ is given by

$$
P_{\mathrm{qs}}^{(\kappa, \alpha)}[\rho(x)] \equiv P_{\mathrm{qs}}^{(\kappa)}[\rho(x)] \simeq \ell^{(\kappa, \alpha)}[\rho(x)] r^{(\kappa, \alpha)}[\rho(x)],
$$

up to normalization constants.

Remark: Note that to obtain both $24 \mathrm{a}$ and $24 \mathrm{c}$ ) we have replaced the sum $\sum_{\mathbf{n} \in \rho(x)} \mathcal{L}(\mathbf{n}) \mathcal{R}(\mathbf{n})$ by $\ell[\rho(x)] \sum_{\mathbf{n} \in \rho(x)} \mathcal{R}(\mathbf{n})$ for large $L$. We will see that this relation is satisfied for the two models that we consider in this paper. It is expected to remain valid for more general diffusive systems [53].

\subsection{Large deviation function}

In our examples we shall see that the hydrodynamic limit of the eigenvectors have a large deviation form given by

$$
\begin{aligned}
& r^{(\kappa, \alpha)}[\rho(x)] \sim e^{-L \psi_{\text {right }}^{(\kappa, \alpha)}[\rho(x)]} \\
& \ell^{(\kappa, \alpha)}[\rho(x)] \sim e^{-L \psi_{\text {left }}^{(\kappa, \alpha)}[\rho(x)]}
\end{aligned}
$$

Using them in (24) gives

$$
\mathcal{P}_{t}^{(\kappa, \alpha)}[\rho(x)] \sim e^{-L \psi_{t}^{(\kappa, \alpha)}[\rho(x)]}
$$

with the large deviation function

- at $t=0$,

$$
\psi_{0}^{(\kappa, \alpha)}[\rho(x)]=\psi_{\text {left }}^{(\kappa, \alpha)}[\rho(x)]+\psi_{\text {right }}^{(0, \alpha)}[\rho(x)]
$$


- at $t=T$,

$$
\psi_{T}^{(\kappa, \alpha)}[\rho(x)]=\psi_{\text {right }}^{(\kappa, \alpha)}[\rho(x)]
$$

- and in the quasi-stationary regime, i.e. $t \gg 1$ and $T-t \gg 1$,

$$
\psi_{t}^{(\kappa, \alpha)}[\rho(x)] \equiv \psi_{\mathrm{qs}}^{(\kappa)}[\rho(x)]=\psi_{\text {left }}^{(\kappa, \alpha)}[\rho(x)]+\psi_{\text {right }}^{(\kappa, \alpha)}[\rho(x)]
$$

where all these equalities are up to an additive constant (independent of $\rho(x)$ ).

Remark: From (15) we see that the large deviation functions in 25 have a simple dependence on $\alpha(x)$, given by

$$
\begin{aligned}
& \psi_{\text {right }}^{(\kappa, \alpha)}[\rho(x)]=V_{\text {right }}^{(\kappa)}[\rho(x)]+\kappa \int_{0}^{1} d x \rho(x) \int_{x}^{1} d y \alpha(y) \\
& \psi_{\text {left }}^{(\kappa, \alpha)}[\rho(x)]=V_{\text {left }}^{(\kappa)}[\rho(x)]-\kappa \int_{0}^{1} d x \rho(x) \int_{x}^{1} d y \alpha(y)
\end{aligned}
$$

where $V_{\text {right }}^{(\kappa)}[\rho(x)]$ and $V_{\text {left }}^{(\kappa)}[\rho(x)]$ are the large deviation functions associated to $\mathcal{R}^{(\kappa)}$ and $\mathcal{L}^{(\kappa)}$ in 15 (following a definition similar to 23 and 25 ).

\section{Independent particles}

In this section, we analyze the simple case of a system of independent particles with transition rates defined in Figure 4. Considering the symmetry 15 it is sufficient to analyze the case $\lambda_{0}=1$, and $\lambda_{i}=0$ for $i \geq 1$. In the rest of our analysis, we shall consider this case, unless explicitly stated otherwise.

In this case the tilted matrix (11) is

$$
\begin{aligned}
{\left[\mathcal{M}_{\kappa} \cdot \Omega\right](\mathbf{n})=} & e^{\kappa} \rho_{a} \Omega\left(n_{1}-1, \ldots\right)+e^{-\kappa}\left(n_{1}+1\right) \Omega\left(n_{1}+1, \ldots\right) \\
& +\left(n_{L}+1\right) \Omega\left(\ldots, n_{L}+1\right)+\rho_{b} \Omega\left(\ldots, n_{L}-1\right) \\
+\sum_{i=1}^{L-1}\left[\left(n_{i}+1\right) \Omega\left(\ldots, n_{i}+1, n_{i+1}-1, \ldots\right)+\left(n_{i+1}+1\right) \Omega\left(\ldots, n_{i}-1, n_{i+1}+1, \ldots\right)\right] & \\
& -\left(\rho_{a}+2 n_{1}+\ldots+2 n_{L}+\rho_{b}\right) \Omega(\mathbf{n})
\end{aligned}
$$

where $\Omega(\mathbf{n})$ is the component of an arbitrary state vector $\boldsymbol{\Omega}$ in the configuration space.

The eigenvalue equations 13 become

$$
\begin{aligned}
& {\left[\mathcal{M}_{\kappa} \cdot \mathcal{R}^{(\kappa)}\right](\mathbf{n})=\mu(\kappa) \mathcal{R}^{(\kappa)}(\mathbf{n})} \\
& {\left[\mathcal{L}^{(\kappa)} \cdot \mathcal{M}_{\kappa}\right](\mathbf{n})=\mu(\kappa) \mathcal{L}^{(\kappa)}(\mathbf{n})}
\end{aligned}
$$

One can check that the right and left eigenvectors are of the form

$$
\begin{aligned}
\mathcal{R}^{(\kappa)}(\mathbf{n}) & =\prod_{i=1}^{L} \frac{a_{i}^{n_{i}}}{n_{i} !} e^{-a_{i}} \\
\mathcal{L}^{(\kappa)}(\mathbf{n}) & =\prod_{i=1}^{L} b_{i}^{n_{i}}
\end{aligned}
$$


where $\left(a_{i}, b_{i}\right)$ are positive numbers to be determined. With this ansatz the eigenvalue equations 29 lead to

$$
\begin{gathered}
e^{-\kappa} a_{1}+a_{L}-\rho_{a}-\rho_{b}-\mu(\kappa)+\sum_{i=1}^{L}\left[e^{\kappa \delta_{i, 1}} \frac{a_{i-1}}{a_{i}}+\frac{a_{i+1}}{a_{i}}-2\right] n_{i}=0 \\
e^{\kappa} \rho_{a} b_{1}+\rho_{b} b_{L}-\rho_{a}-\rho_{b}-\mu(\kappa)+\sum_{i=1}^{L}\left[e^{-\kappa \delta_{i, 1}} \frac{b_{i-1}}{b_{i}}+\frac{b_{i+1}}{b_{i}}-2\right] n_{i}=0
\end{gathered}
$$

with $a_{0}=\rho_{a}, a_{L+1}=\rho_{b}$, and $b_{0}=1=b_{L+1}$. As the occupation variables $n_{i}$ are arbitrary, for the equations to be satisfied, their coefficients must vanish. This leads to a set of coupled linear equations for $a_{i}$ and $b_{i}$, which can be easily solved, and we get, for $1 \leq i \leq L$,

$$
\begin{aligned}
a_{i} & =\rho_{a}\left(1-\frac{i}{L+1}\right) e^{\kappa}+\rho_{b} \frac{i}{L+1} \\
b_{i} & =\left(1-\frac{i}{L+1}\right) e^{-\kappa}+\frac{i}{L+1}
\end{aligned}
$$

with the largest eigenvalue

$$
\mu(\kappa)=\mu_{\mathrm{ni}}(\kappa)=\frac{\rho_{a}}{L+1}\left(e^{\kappa}-1\right)+\frac{\rho_{b}}{L+1}\left(e^{-\kappa}-1\right)
$$

\subsection{Hydrodynamic limit}

In the hydrodynamic limit, from $(32)$ it is easy to confirm 20, which gives

$$
\chi(\kappa) \equiv \chi_{\mathrm{ni}}(\kappa)=\rho_{a}\left(e^{\kappa}-1\right)+\rho_{b}\left(e^{-\kappa}-1\right)
$$

For the hydrodynamic limit of the right eigenvector, defined in $23 \mathrm{a}$, we decompose the system into $M=L / w$ boxes each containing $w$ sites and define

$$
r^{(\kappa)}\left(\rho_{1}, \ldots, \rho_{M}\right)=\sum_{\mathbf{n}} \mathcal{R}^{(\kappa)}(\mathbf{n})
$$

where the sum is over all configurations with $\rho_{m} w$ particles in the $m$-th box. Then, using (30a) we get

$r^{(\kappa)}\left(\rho_{1}, \ldots, \rho_{M}\right)=\prod_{m=1}^{M}\left\{\sum_{n_{m_{1}}} \cdots \sum_{n_{m_{w}}}\left[\frac{a_{m_{1}}^{n_{m_{1}}}}{n_{m_{1}} !} \cdots \frac{a_{m_{w}}^{n_{m_{w}}}}{n_{m_{w}} !} e^{-a_{m_{1}} \cdots-a_{m_{w}}}\right] \delta_{\rho_{m} w, \sum_{i} n_{m_{i}}}\right\}$

where $m_{i}$ denotes the site index of the $i$-th site of the $m$ th box, and $\delta_{i, j}$ is the Kronecker delta. Using an identity

$$
\sum_{n_{1}=0}^{\infty} \sum_{n_{2}=0}^{\infty} \frac{a_{1}^{n_{1}}}{n_{1} !} \frac{a_{2}^{n_{2}}}{n_{2} !} \delta_{n, n_{1}+n_{2}}=\frac{\left(a_{1}+a_{2}\right)^{n}}{n !}
$$


and that $a_{i}$ is slowly varying such that $a_{i} \simeq a\left(\frac{m w}{L}\right)$ when the site $i$ is in the $m$-th box, and defining $\rho_{m}=\rho\left(\frac{m w}{L}\right)$ we get

$$
r^{(\kappa)}\left(\rho_{1}, \ldots, \rho_{M}\right) \simeq \prod_{m=1}^{M} \frac{\left[w a\left(\frac{m w}{L}\right)\right]^{w \rho\left(\frac{m w}{L}\right)}}{\left[w \rho\left(\frac{m w}{L}\right)\right] !} e^{-w a\left(\frac{m w}{L}\right)}
$$

Then, for $1 \ll w \ll L$, using the Stirling's formula we get (following the definition 23a, 25a 28a)

$$
r^{(\kappa)}\left(\rho_{1}, \ldots, \rho_{M}\right) \simeq r^{(\kappa)}[\rho(x)] \sim e^{-L V_{\text {right }}^{(\kappa)}[\rho(x)]}
$$

with

$$
V_{\text {right }}^{(\kappa)}[\rho(x)]=H_{\mathrm{ni}}[\rho(x), a(x)]
$$

where we defined

$$
H_{\mathrm{ni}}[\rho(x), a(x)]=\int_{0}^{1} d x\left[\rho(x) \log \frac{\rho(x)}{a(x)}-\rho(x)+a(x)\right]
$$

and

$$
a(x)=\rho_{a}(1-x) e^{\kappa}+\rho_{b} x
$$

For the left eigenvector, the hydrodynamic limit $23 \mathrm{~b}$ is simple to construct from $(30 \mathrm{~b})$, which (following the definition $23 \mathrm{~b}, 25 \mathrm{~b}, 28 \mathrm{~b})$ ) leads to $\ell^{(\kappa)}[\rho(x)] \sim$ $e^{-L V_{\text {left }}^{(\kappa)}[\rho(x)]}$ with

$$
V_{\text {left }}^{(\kappa)}[\rho(x)]=\int_{0}^{1} d x \rho(x) \log \frac{1}{b(x)}
$$

and

$$
b(x)=e^{-\kappa}(1-x)+x
$$

Remark: The $\kappa=0$ corresponds to the case without a condition on the empirical observable. So, the steady state large deviation function of density $\mathcal{F}_{\text {ni }}[\rho(x)]=$ $V_{\text {right }}^{(0)}[\rho(x)]$ is

$$
\mathcal{F}_{\text {ni }}[\rho(x)]=H_{\text {ni }}\left[\rho(x), \bar{\rho}_{\text {free }}(x)\right] \quad \text { with } \quad \bar{\rho}_{\text {free }}(x)=\rho_{a}(1-x)+\rho_{b} x
$$


3.2 Large deviation function

Expressions for $\psi_{\text {left }}^{(\kappa, \alpha)}$ and $\psi_{\text {right }}^{(\kappa, \alpha)}$ for an arbitrary $\alpha(x)$ follow from 28 . Using this result in 27) we get the large deviation function at three different times, all of which are of the form

$$
\psi_{t}^{(\kappa, \alpha)}[\rho(x)]=H_{\mathrm{ni}}\left[\rho(x), \bar{\rho}_{t}^{(\kappa, \alpha)}(x)\right]
$$

with the average density profile

1. at $t=0$,

$$
\bar{\rho}_{0}^{(\kappa, \alpha)}(x)=\bar{\rho}_{\text {free }}(x)\left[(1-x) e^{-\kappa \int_{0}^{x} d y \alpha(y)}+x e^{\kappa \int_{x}^{1} d y \alpha(y)}\right]
$$

2. at $t=T$,

$$
\bar{\rho}_{T}^{(\kappa, \alpha)}(x)=\rho_{a}(1-x) e^{\kappa \int_{0}^{x} d y \alpha(y)}+\rho_{b} x e^{-\kappa \int_{x}^{1} d y \alpha(y)}
$$

3. and in the quasi-stationary regime,

$$
\bar{\rho}_{\mathrm{qS}}(x)=\bar{\rho}_{\text {free }}(x)+x(1-x)\left[\rho_{a}\left(e^{\kappa}-1\right)+\rho_{b}\left(e^{-\kappa}-1\right)\right]
$$

\section{Symmetric simple exclusion process}

In this section, we analyze the one-dimensional symmetric simple exclusion process with the transition rates defined in Figure 5. We indicate how to perform a low-density expansion. Our results will be limited to the first two terms in this expansion, although it is straightforward to extend our approach to higher orders. Considering the symmetry 15 we will analyze only the case $\lambda_{0}=1$, and $\lambda_{i}=0$ for $i \geq 1$.

\subsection{A representation of the eigenvectors}

A configuration can be specified by the position of the particles. In a configuration with $m$ occupied sites $\left\{i_{1}, \ldots, i_{m}\right\}$ we denote the component of the eigenvectors as

$$
\mathcal{R}^{(\kappa)}(\mathbf{n}) \equiv \mathcal{R}^{(\kappa)}\left(i_{1}, \ldots, i_{m}\right) \quad \text { and } \quad \mathcal{L}^{(\kappa)}(\mathbf{n}) \equiv \mathcal{L}^{(\kappa)}\left(i_{1}, \ldots, i_{m}\right)
$$

We normalize such that the component of both eigenvectors in the empty configuration is 1 .

\subsection{A perturbation solution for small density}

For finite $L$, the Perron-Frobenius theorem [54] assures that the largest eigenvalue of the tilted matrix is non-degenerate. Expressions of the eigenvalue and eigenvectors exist [55, 56], but it is hard to extract from them the large scale behaviors. 
Here, we use a perturbation expansion in small $\rho_{a}$ and $\rho_{b}$, where it is possible to systematically solve the eigenvalue equation order by order. We write

$$
\begin{aligned}
& \mu(\kappa)=\mu_{0}(\kappa)+\mu_{1}(\kappa)+\mu_{2}(\kappa)+\cdots \\
& \mathcal{R}^{(\kappa)}=\mathcal{R}_{0}^{(\kappa)}+\mathcal{R}_{1}^{(\kappa)}+\mathcal{R}_{2}^{(\kappa)}+\cdots \\
& \mathcal{L}^{(\kappa)}=\mathcal{L}_{0}^{(\kappa)}+\mathcal{L}_{1}^{(\kappa)}+\mathcal{L}_{2}^{(\kappa)}+\cdots
\end{aligned}
$$

with increasing orders in $\rho_{a}$ and $\rho_{b}$.

For $\rho_{a}=\rho_{b}=0$, all $\mathcal{R}_{n}^{(\kappa)}=0$ except for the empty configuration and one has $\mu_{0}(\kappa)=0$. It is also clear that at order $n$ in $\rho_{a}$ and $\rho_{b}$, the $\mathcal{R}_{n}^{(\kappa)}$ of configurations with more than $n$ occupied sites vanish. Therefore,

$$
\begin{aligned}
& \mathcal{R}_{0}^{(\kappa)}(i)=0, \\
& \mathcal{R}_{0}^{(\kappa)}(i, j)=\mathcal{R}_{1}^{(\kappa)}(i, j)=0, \\
& \mathcal{R}_{0}^{(\kappa)}(i, j, k)=\mathcal{R}_{1}^{(\kappa)}(i, j, k)=\mathcal{R}_{2}^{(\kappa)}(i, j, k)=0,
\end{aligned}
$$

and so on. Here we present the solution up to only the second order.

The equations one needs to solve up to the second order in $\rho_{a}$ and $\rho_{b}$ are given in the Appendix A. Since at this order $\mathcal{R}_{2}^{(\kappa)}$ of configurations with 3 or more occupied sites vanish, the hierarchy closes. For arbitrary $L$, we get the solution

$$
\begin{aligned}
& \mu_{1}(\kappa)=\frac{\rho_{a}}{L+1}\left(e^{\kappa}-1\right)+\frac{\rho_{b}}{L+1}\left(e^{-\kappa}-1\right) \\
& \mu_{2}(\kappa)=-\frac{\left(e^{\kappa}-1\right)^{2}}{6(L+1)^{2}}\left[2 L\left(\rho_{a}^{2}+\rho_{a} \rho_{b} e^{-\kappa}+\rho_{b}^{2} e^{-2 \kappa}\right)+\rho_{a}^{2}+4 \rho_{a} \rho_{b} e^{-\kappa}+\rho_{b}^{2} e^{-2 \kappa}\right]
\end{aligned}
$$

in agreement with an earlier result [57. The right eigenvector, up to the second order, is given by

$$
\begin{aligned}
\mathcal{R}_{1}^{(\kappa)}(i)= & e^{\kappa} \rho_{a}\left(1-\frac{i}{L+1}\right)+\rho_{b} \frac{i}{L+1} \\
\mathcal{R}_{2}^{(\kappa)}(i)= & e^{\kappa} \rho_{a}^{2}\left(1-\frac{i}{L+1}\right)+\rho_{b}^{2} \frac{i}{L+1} \\
& -\left(e^{\kappa} \rho_{a}-\rho_{b}\right)^{2} \frac{i}{6 L}\left(1-\frac{i}{L+1}\right)\left[e^{-\kappa}\left(2-\frac{i}{L+1}\right)+1+\frac{i}{L+1}\right] \\
\mathcal{R}_{2}^{(\kappa)}(i, j)- & \mathcal{R}_{1}^{(\kappa)}(i) \mathcal{R}_{1}^{(\kappa)}(j)=-\frac{\left(\rho_{a} e^{\kappa}-\rho_{b}\right)^{2}}{(L+1)} \frac{i}{L}\left(1-\frac{j}{L+1}\right) \quad \text { for } j \geq i
\end{aligned}
$$

As the probabilities (14) are always a product of a left and a right eigenvector, we will only need the following orders for the left eigenvector.

$$
\begin{aligned}
& \mathcal{L}_{0}^{(\kappa)}(i)=e^{-\kappa}\left(1-\frac{i}{L+1}\right)+\frac{i}{L+1} \\
& \mathcal{L}_{1}^{(\kappa)}(i)=\left(e^{-\kappa}-1\right)^{2} \frac{i}{6 L}\left(\frac{i}{L+1}-1\right)\left[e^{\kappa} \rho_{a}\left(2-\frac{i}{L+1}\right)+\rho_{b}\left(1+\frac{i}{L+1}\right)\right] \\
& \mathcal{L}_{0}^{(\kappa)}(i, j)-\mathcal{L}_{0}^{(\kappa)}(i) \mathcal{L}_{0}^{(\kappa)}(j)=-\frac{\left(e^{-\kappa}-1\right)^{2}}{(L+1)} \frac{i}{L}\left(1-\frac{j}{L+1}\right) \quad \text { for } j \geq i
\end{aligned}
$$




\section{Remarks:}

- For the symmetric simple exclusion process, the eigenvalue equation can be systematically solved to arbitrary order. The "miracle" which makes an explicit solution possible in practice is that at every order, the eigenvectors are low degree polynomials of the site indices $i$. This is special to the exclusion process, and may not apply to other diffusive systems.

- At the first order in $\rho_{a}$ and $\rho_{b}$, the expressions of eigenvalue and eigenvectors coincide with that of the independent particles case in Section 3.

\subsection{Cumulants of the occupation variable}

One can then derive the cumulants of the occupation variables from the probability (14). For example, at time $\tau=N$, using (14b) and the representation (39) we get

$$
\begin{aligned}
\left\langle n_{i}\right\rangle & =\frac{1}{\mathcal{N}}\left(\mathcal{R}^{(\kappa)}(i)+\sum_{j \neq i} \mathcal{R}^{(\kappa)}(i, j)+\cdots\right) \\
\left\langle n_{i} n_{j}\right\rangle & =\frac{1}{\mathcal{N}}\left(\mathcal{R}^{(\kappa)}(i, j)+\sum_{k \neq i, j} \mathcal{R}^{(\kappa)}(i, j, k)+\cdots\right)
\end{aligned}
$$

where the normalization $\mathcal{N}=1+\sum_{i} \mathcal{R}^{(\kappa)}(i)+\sum_{i} \sum_{j \neq i} \mathcal{R}^{(\kappa)}(i, j)+\cdots$.

Using this with the perturbation solution of the eigenvectors we can construct a perturbation expansion of the cumulants for the low density limit. For example, at the second order in $\rho_{a}$ and $\rho_{b}$, the average occupation of a site $i$ at time $\tau=N$ is

$$
\left\langle n_{i}\right\rangle=\mathcal{R}_{1}^{(\kappa)}(i)+\mathcal{R}_{2}^{(\kappa)}(i)-\left(\mathcal{R}_{1}^{(\kappa)}(i)\right)^{2}+\sum_{j \neq i}\left(\mathcal{R}_{2}^{(\kappa)}(i, j)-\mathcal{R}_{1}^{(\kappa)}(i) \mathcal{R}_{1}^{(\kappa)}(j)\right)+\cdots
$$

and the connected correlation

$$
\left\langle n_{i} n_{j}\right\rangle_{c}=\mathcal{R}_{2}^{(\kappa)}(i, j)-\mathcal{R}_{1}^{(\kappa)}(i) \mathcal{R}_{1}^{(\kappa)}(j)+\cdots
$$

For time $\tau=0$ and for the quasi-stationary state (see $(14)$ ) we can similarly write the average occupations and their correlations by replacing $\mathcal{R}^{(\kappa)}$ by $\mathcal{R}^{(0)} \mathcal{L}^{(\kappa)}$ or $\mathcal{R}^{(\kappa)} \mathcal{L}^{(\kappa)}$ in (44).

\subsection{Hydrodynamic limit}

It is straightforward to take the hydrodynamic limit of the expressions 42 and (43), which can be used to derive the hydrodynamic limit of the cumulants of the occupation variables. For large $L$, we define

$$
\begin{aligned}
\ell^{(\kappa)}(x) & \simeq \mathcal{L}_{0}^{(\kappa)}(x L)+\mathcal{L}_{1}^{(\kappa)}(x L) \\
& =e^{-\kappa}(1-x)+x-\frac{1}{6}\left(e^{-\kappa}-1\right)^{2} x(1-x)\left[e^{\kappa} \rho_{a}(2-x)+\rho_{b}(1+x)\right]
\end{aligned}
$$


and

$$
\begin{aligned}
g_{\text {left }}^{(\kappa)}(x, y) & \simeq L\left[\mathcal{L}_{0}^{(\kappa)}(x L, y L)-\mathcal{L}_{0}^{(\kappa)}(x L) \mathcal{L}_{0}^{(\kappa)}(y L)\right] \\
& =-\left(e^{-\kappa}-1\right)^{2} x(1-y) \quad \text { for } y \geq x .
\end{aligned}
$$

Similarly, from 42 we define

$$
\begin{aligned}
r^{(\kappa)}(x) & \simeq \mathcal{R}_{1}^{(\kappa)}(x L)+\mathcal{R}_{2}^{(\kappa)}(x L) \\
= & e^{\kappa}\left(\rho_{a}+\rho_{a}^{2}\right)(1-x)+\left(\rho_{b}+\rho_{b}^{2}\right) x \\
& \quad-\frac{1}{6}\left(e^{\kappa} \rho_{a}-\rho_{b}\right)^{2} x(1-x)\left[1+x+(2-x) e^{-\kappa}\right]
\end{aligned}
$$

and

$$
\begin{aligned}
g_{\text {right }}^{(\kappa)}(x, y) & \simeq L\left[\mathcal{R}_{2}^{(\kappa)}(x L, y L)-\mathcal{R}_{1}^{(\kappa)}(x L) \mathcal{R}_{1}^{(\kappa)}(y L)\right] \\
& =-\left(e^{\kappa} \rho_{a}-\rho_{b}\right)^{2} x(1-y) \quad \text { for } y \geq x .
\end{aligned}
$$

Remark: In taking the hydrodynamic limit of the perturbation expansion we have assumed that the limits of large $L$ and small density can be exchanged. We will see that the resulting large deviation functions are consistent with the macroscopic analysis in Section 7.2 .

\subsubsection{Cumulants of density}

At time $\tau=0$, in the quasi-stationary regime, and at $\tau=L^{2} T$ (the hydrodynamic time $t=T$ ) the cumulants of the occupation variable are of the form (see (44))

$$
\begin{aligned}
\left\langle n_{x L}\right\rangle & \simeq \bar{\rho}(x)=u(x)[1-u(x)]+\int_{0}^{1} d y c(x, y) \\
\left\langle n_{x L} n_{y L}\right\rangle_{c} & \simeq \frac{1}{L} c(x, y)
\end{aligned}
$$

for large $L$, up to the second order in $\rho_{a}$ and $\rho_{b}$, where

- at $t=0$,

$$
\begin{aligned}
u(x) & =\ell^{(\kappa)}(x) r^{(0)}(x) \\
c(x, y) & =\ell^{(\kappa)}(x) \ell^{(\kappa)}(y) g_{\text {right }}^{(0)}(x, y)+r^{(0)}(x) r^{(0)}(y) g_{\text {left }}^{(\kappa)}(x, y)
\end{aligned}
$$

- in the quasi-stationary state,

$$
\begin{aligned}
u(x) & =\ell^{(\kappa)}(x) r^{(\kappa)}(x) \\
c(x, y) & =\ell^{(\kappa)}(x) \ell^{(\kappa)}(y) g_{\text {right }}^{(\kappa)}(x, y)+r^{(\kappa)}(x) r^{(\kappa)}(y) g_{\text {left }}^{(\kappa)}(x, y)
\end{aligned}
$$

- at $t=T$,

$$
u(x)=r^{(\kappa)}(x) \quad \text { and } \quad c(x, y)=g_{\text {right }}^{(\kappa)}(x, y)
$$

Explicit expressions of the cumulants are given in Appendix B. 
4.4.2 Large deviation function

Here, we show how to derive a small density expansion of the large deviation function in 26 from the above expansion of the cumulants of the density.

Take an arbitrary probability distribution $P(\mathbf{n})$ where the occupation variables are either 0 or 1 . Then, one can write

$$
\prod_{i=1}^{L} e^{h_{i} n_{i}}=\prod_{i=1}^{L}\left[1+n_{i}\left(e^{h_{i}}-1\right)\right]
$$

where $h_{i}$ is a real valued parameter. If one expands this product, averages over $P(\mathbf{n})$, and uses the fact that cumulants of order $k$ scale like $\rho_{a}$ and $\rho_{b}$ to the power $k$, then one gets, for finite $L$,

$$
\begin{aligned}
\log \left\langle\prod_{i=1}^{L} e^{h_{i} n_{i}}\right\rangle= & \sum_{i=1}^{L}\left\langle n_{i}\right\rangle\left(e^{h_{i}}-1\right)-\frac{1}{2} \sum_{i=1}^{L}\left\langle n_{i}\right\rangle^{2}\left(e^{h_{i}}-1\right)^{2} \\
& +\sum_{i=1}^{L} \sum_{j>i}^{L}\left\langle n_{i} n_{j}\right\rangle_{c}\left(e^{h_{i}}-1\right)\left(e^{h_{j}}-1\right)+\cdots
\end{aligned}
$$

In the hydrodynamic limit, when $h_{i} \simeq h\left(\frac{i}{L}\right),\left\langle n_{i}\right\rangle \simeq \bar{\rho}\left(\frac{i}{L}\right)$, and $\left\langle n_{i} n_{j}\right\rangle_{c} \simeq$ $\frac{1}{L} c\left(\frac{i}{L}, \frac{j}{L}\right)$, we get for the generating functional

$$
\log \left\langle\prod_{i=1}^{L} e^{h_{i} n_{i}}\right\rangle \simeq L \mathcal{G}[h(x)]
$$

with

$$
\begin{aligned}
\mathcal{G}[h(x)]= & \int_{0}^{1} d x\left(e^{h(x)}-1\right) \bar{\rho}(x)-\frac{1}{2} \int_{0}^{1} d x\left(e^{h(x)}-1\right)^{2} \bar{\rho}(x)^{2} \\
& +\int_{0}^{1} d x \int_{x}^{1} d y\left(e^{h(x)}-1\right)\left(e^{h(y)}-1\right) c(x, y)+\cdots
\end{aligned}
$$

Then, $P[\rho(x)]$ has [7, 35 the large deviation form $(26)$ with the large deviation function $\psi[\rho(x)]$ given by the Legendre transformation

$$
\psi[\rho(x)]=\int_{0}^{1} d x h(x) \rho(x)-\mathcal{G}[h(x)]
$$

where $h(x)$ is the solution of $\frac{\delta \mathcal{G}[h]}{\delta h(x)}=\rho(x)$.

The small density expansion of (53,54) is then straightforward to get from the perturbation expansion of the cumulants. It gives

$$
\psi[\rho(x)] \simeq H_{\mathrm{sep}}[\rho(x), \bar{\rho}(x), c(x, y)]
$$

where up to the second order in $\rho_{a}$ and $\rho_{b}$,

$$
\begin{aligned}
H_{\mathrm{sep}}[\rho(x), \bar{\rho}(x), c(x, y)]= & \int_{0}^{1} d x\left[\rho(x) \log \frac{\rho(x)}{\bar{\rho}(x)}+(1-\rho(x)) \log \frac{1-\rho(x)}{1-\bar{\rho}(x)}\right] \\
& -\frac{1}{2} \int_{0}^{1} d x \int_{0}^{1} d y\left(\frac{\rho(x)}{\bar{\rho}(x)}-1\right)\left(\frac{\rho(y)}{\bar{\rho}(y)}-1\right) c(x, y)
\end{aligned}
$$


The expression (55) remains valid at all times with $\bar{\rho}(x)$ and $c(x, y)$ being replaced by their expressions in Section 4.4.1. For the unconditioned case, where $\bar{\rho}(x)$ and $c(x, y)$ are given by 49 for $\kappa=0$, one can verify that this perturbation expansion result is consistent with previously known expressions [35, 39, 43.

4.4.3 Small density expansion of $\chi(\kappa), V_{\text {left }}^{(\kappa)}$ and $V_{\text {right }}^{(\kappa)}$

It is straightforward to take the hydrodynamic limit of (41), which confirms 20 at the level of a small density expansion with

$$
\chi(\kappa)=\rho_{a}\left(e^{\kappa}-1\right)+\rho_{b}\left(e^{-\kappa}-1\right)-\frac{1}{3}\left(1-e^{-\kappa}\right)^{2}\left(e^{2 \kappa} \rho_{a}^{2}+e^{\kappa} \rho_{a} \rho_{b}+\rho_{b}^{2}\right)+\cdots
$$

This is in agreement with earlier findings in [41, 51].

For $\lambda_{0}=1$ and $\lambda_{i}=0$ for $i \geq 1$, the large deviation functions $\psi_{\text {left }}^{(\kappa, \alpha)} \equiv V_{\text {left }}^{(\kappa)}$ and $\psi_{\text {right }}^{(\kappa, \alpha)} \equiv V_{\text {right }}^{(\kappa)}($ see $(28))$. An expression for $V_{\text {left }}^{(\kappa)}$ can be derived by taking the hydrodynamic limit $23 \mathrm{~b}, 25 \mathrm{~b}$ of $\mathcal{L}^{(\kappa)}$. For a configuration $\mathbf{n}$, where the occupation variables are either 0 or 1 , we write

$$
\log \mathcal{L}^{(\kappa)}=\sum_{i} n_{i} \log \mathcal{L}^{(\kappa)}(i)+\sum_{i<j} n_{i} n_{j} \log \left[1+\frac{\mathcal{L}^{(\kappa)}(i, j)-\mathcal{L}^{(\kappa)}(i) \mathcal{L}^{(\kappa)}(j)}{\mathcal{L}^{(\kappa)}(i) \mathcal{L}^{(\kappa)}(j)}\right]+\cdots
$$

using the representation in (39). Then, from the perturbation expansion 40c 43) and taking the hydrodynamic limit $23 \mathrm{~b}, 25 \mathrm{~b}$ we get

$$
V_{\text {left }}^{(\kappa)}[\rho]=\int_{0}^{1} d x \rho(x) \log \frac{1}{\ell^{(\kappa)}(x)}-\frac{1}{2} \int_{0}^{1} d x \int_{0}^{1} d y \frac{\rho(x) \rho(y)}{\ell^{(\kappa)}(x) \ell^{(\kappa)}(y)} g_{\text {left }}^{(\kappa)}(x, y)+\cdots
$$

where $\ell^{(\kappa)}(x)$ and $g_{\text {left }}^{(\kappa)}(x, y)$ are defined in 45 and 46$)$.

In comparison, it is harder to derive an expression for $V_{\text {right }}^{(\kappa)}$ by taking the hydrodynamic limit of the expression 42 for $\mathcal{R}^{(\kappa)}$. It is much easier to derive using the relation $(27 \mathrm{~b})$ and the result $49 \sqrt[55]{5}$ at time $T$. This gives

$$
\begin{aligned}
V_{\text {right }}^{(\kappa)}[\rho(x)] & =H_{\text {sep }}\left[\rho(x), \bar{\rho}_{T}(x), g_{\text {right }}^{(\kappa)}(x, y)\right] \\
\text { with } \quad \bar{\rho}_{T}(x) & =r^{(\kappa)}(x)\left(1-r^{(\kappa)}(x)\right)+\int_{0}^{1} d y g_{\text {right }}^{(\kappa)}(x, y)
\end{aligned}
$$

up to the second order in $\rho_{a}$ and $\rho_{b}$, and an additive constant.

Remark: We have checked (details in Appendix C) that the result (57), and the expression of $\psi_{\mathrm{qs}}^{(\kappa)}$ and $\psi_{T}^{(\kappa)}$ obtained from (55) satisfy the relation $27 \mathrm{c}$.

\section{Effective dynamics}

In the canonical ensemble, the biased dynamics is Markovian and one can write [8, 22, 28, the transition rates in terms of the tilted Matrix. For example, in 
the quasi-stationary regime, the transition rate $W_{\mathrm{qs}}^{(\kappa, \boldsymbol{\lambda})}\left(\mathbf{n}^{\prime}, \mathbf{n}\right)$ from a microscopic configuration $\mathbf{n}$ to another configuration $\mathbf{n}^{\prime}$ is given by [28]

$$
W_{\mathrm{qS}}^{(\kappa, \boldsymbol{\lambda})}\left(\mathbf{n}^{\prime}, \mathbf{n}\right)=\frac{\mathcal{L}^{(\kappa, \boldsymbol{\lambda})}\left(\mathbf{n}^{\prime}\right)}{\mathcal{L}^{(\kappa, \boldsymbol{\lambda})}(\mathbf{n})} \mathcal{M}_{\kappa}^{(\boldsymbol{\lambda})}\left(\mathbf{n}^{\prime}, \mathbf{n}\right) \quad \text { for } \mathbf{n}^{\prime} \neq \mathbf{n} .
$$

This means, a spontaneous fluctuation at time $\tau_{0}$ in the quasi-stationary regime $\left(1 \ll \tau_{0}\right.$ and $\left.1 \ll N-\tau_{0}\right)$, relaxes following this dynamics $59 \mathrm{a}$ ).

Similarly, the path leading to a spontaneous fluctuation can be described by a time reversal of 59a. The transition rate $\mathbb{W}_{\mathrm{qs}}^{(\kappa, \boldsymbol{\lambda})}\left(\mathbf{n}^{\prime}, \mathbf{n}\right)$ of this time-reversed process can be constructed [58] using the quasi-stationary distribution [14c], which gives

$$
\mathbb{W}_{\mathrm{qs}}^{(\kappa, \boldsymbol{\lambda})}\left(\mathbf{n}^{\prime}, \mathbf{n}\right)=\frac{\mathcal{R}^{(\kappa, \boldsymbol{\lambda})}\left(\mathbf{n}^{\prime}\right)}{\mathcal{R}^{(\kappa, \boldsymbol{\lambda})}(\mathbf{n})} \mathcal{M}_{\kappa}^{(\boldsymbol{\lambda})}\left(\mathbf{n}, \mathbf{n}^{\prime}\right) \quad \text { for } \mathbf{n}^{\prime} \neq \mathbf{n}
$$

For the two examples considered in this paper (the independent particles and the symmetric simple exclusion process), it is straightforward to see that the effective dynamics (59a) with (11) correspond to re-weighting the jump rates of particles (see Figure 4 and Figure 5): the jump rate for a particle from site $i$ to $i+1$ is weighted by a factor $e^{E_{i}}$, whereas the jump rate from $i+1$ to $i$ is weighted by $e^{-E_{i}}$, where

$$
E_{i}(\mathbf{n})=\kappa \lambda_{i}+\log \frac{\mathcal{L}^{(\kappa, \boldsymbol{\lambda})}(\widehat{\mathbf{n}})}{\mathcal{L}^{(\kappa, \boldsymbol{\lambda})}(\mathbf{n})} \quad \text { for all } 0 \leq i \leq L
$$

with

$$
\widehat{n}_{j}=n_{j}-\delta_{j, i}+\delta_{j, i+1} \quad \text { for all } 1 \leq j \leq L .
$$

Similar re-weighting of jump rates can be seen for the time reversed dynamics (59b). We note that, in general, the jump rates for the dynamics are non-local functions of the occupation variables $\mathbf{n}$.

\section{Hydrodynamic limit}

In the large $L$ limit, when $x=\frac{i}{L}$ and $\lambda_{i}=\frac{1}{L} \alpha(x), 60$ becomes

$$
E_{i}(\mathbf{n}) \simeq \frac{1}{L} e(x) \quad \text { with } \quad e(x)=\kappa \alpha(x)-\partial_{x} \frac{\delta \psi_{\text {left }}^{(\kappa, \alpha)}}{\delta \rho(x)} .
$$

In a hydrodynamic description (1), the effect of such a weak bias can be incorporated (see [59] for another example) within the linear response theory, where $e(x)$ acts as an external driving field. This leads to the following dynamics.

Relaxation: A spontaneous fluctuation of hydrodynamic density in the quasistationary state relaxes following $\partial_{t} \rho(x, t)=-\partial_{x} j(x, t)$ with

$$
j(x, t)=-D(\rho(x, t)) \partial_{x} \rho(x, t)+\sigma(\rho(x, t))\left\{\kappa \alpha(x)-\partial_{x} \frac{\delta \psi_{\text {left }}^{(\kappa, \alpha)}}{\delta \rho(x, t)}\right\}+\eta(x, t)
$$

where $\eta(x, t)$ is a Gaussian white noise of zero mean and covariance (1b).

A somewhat similar analysis based on $59 \mathrm{~b}$ leads to the following dynamics: 
Fluctuation: The path leading to a fluctuation in the quasi-stationary state is described by $\partial_{t} \rho(x, t)=-\partial_{x} j(x, t)$ with

$$
j(x, t)=-D(\rho(x, t)) \partial_{x} \rho(x, t)+\sigma(\rho(x, t))\left\{\kappa \alpha(x)+\partial_{x} \frac{\delta \psi_{\text {right }}^{(\kappa, \alpha)}}{\delta \rho(x, t)}\right\}+\eta(x, t)
$$

Remark: The time evolution of the most probable density profile leading to a fluctuation and its subsequent relaxation are the zero noise case of $(62 \mathrm{~b})$ and $62 \mathrm{a})$. We have verified this explicitly for the independent particles starting from their microscopic dynamics. For the symmetric simple exclusion process, we checked this up to the second order in a low density expansion.

\section{Macroscopic analysis}

The two examples discussed in Section 3 to Section 5 are governed [35, 38, 41, 60, for large $L$, by the fluctuating hydrodynamics equation (1). Our goal here is to show that the large deviation functions $(34,35)$ and $(57,58)$ are consistent with a macroscopic approach. Besides this, the macroscopic analysis applies for a general class of models where the microscopic details enter in the terms $D(\rho)$ and $\sigma(\rho)$.

Much of the results can be infered by drawing an analogy of (1) to a Langevin equation in the weak noise limit, as in [28. In this analogy, a simple quantity is the generating function, which for (1) is defined by

$$
G_{T}^{(\kappa, \alpha)}[r(x), s(x)]=\int d Q e^{L \kappa Q} P_{T}^{(\alpha)}[r(x), Q \mid s(x)]
$$

where $P_{T}^{(\alpha)}[r(x), Q \mid s(x)]$ is the joint probability of a density profile $\rho(x, T)=r(x)$ at the hydrodynamic time $t=T$, and $Q_{T}^{(\alpha)}$ in 3 to take value $Q$ given the density $\rho(x, 0)=s(x)$ at $t=0$. Similarly to the Langevin equation [28], one expects, for large $L$ and $T$, the generating function to have the form

$$
G_{T}^{(\kappa, \alpha)}[r(x), s(x)] \sim e^{T L \chi(\kappa)-L \psi_{\mathrm{right}}^{(\kappa, \alpha)}[r(x)]-L \psi_{\text {left }}^{(\kappa, \alpha)}[s(x)]}
$$

where $\chi(\kappa), \psi_{\text {right }}^{(\kappa, \alpha)}$, and $\psi_{\text {left }}^{(\kappa, \alpha)}$ are the same quantities as in 20) and 25). Starting from (64) we now obtain a variational formulation as in 33 35, 39.

\subsection{A variational formulation}

For large $L$, the probability of a certain time evolution of $\rho(x, t)$ and $j(x, t)$ inside the time window $[0, T]$, which follows $(1)$ is given by [33, 35.

$$
P[\rho(x, t), j(x, t)] \sim \exp \left[-L \int_{0}^{1} d x \int_{0}^{T} d t \frac{\left(j(x, t)+D(\rho) \partial_{x} \rho(x, t)\right)^{2}}{2 \sigma(\rho)}\right]
$$

where $\sim$ means that sub-leading terms in large $L$ are neglected. Using this, the generating function 63 can be written as a path-integral

$$
G_{T}^{(\kappa, \alpha)}[r(x), s(x)] \sim \int \mathcal{D}[\rho, j] e^{L S_{T}^{(\kappa, \alpha)}[\rho, j]}
$$


with

$$
S_{t_{f}-t_{i}}^{(\kappa, \alpha)}[\rho, j]=\int_{t_{i}}^{t_{f}} d t \int_{0}^{1} d x\left\{\kappa \alpha(x) j(x, t)-\frac{\left(j(x, t)+D(\rho) \partial_{x} \rho(x, t)\right)^{2}}{2 \sigma(\rho)}\right\}
$$

with $t_{i}=0$ and $t_{f}=T$. The path integral in 66a is over all paths $\{\rho(x, t), j(x, t)\}$ satisfying $\partial_{t} \rho=-\partial_{x} j$ with the initial density profile $\rho(x, 0)=s(x)$ and the final density profile $\rho(x, T)=r(x)$.

For large $L$ and $T$, assuming a single optimal path, we get the large deviation form 64 with

$$
T \chi(\kappa)-\psi_{\text {right }}^{(\kappa, \alpha)}[r(x)]-\psi_{\text {left }}^{(\kappa, \alpha)}[s(x)]=\max _{\rho, j} S_{T}^{(\kappa, \alpha)}[\rho, j]
$$

where the optimization is over all paths $(\rho(x, t), j(x, t))$ satisfying the conditions mentioned earlier.

In a rather general class of systems [48, the optimal path for (67) starts at the given density profile $\rho(x, 0)=s(x)$ but soon it becomes time independent $\rho(x, t)=\bar{\rho}_{\mathrm{qs}}(x)$ and remains at this density until only close to the final time $T$ where it changes to $\rho(x, T)=r(x)$. (This assumption for the time independence of the optimal profile for $t \gg 1$ and $T-t \gg 1$ is equivalent to assuming the additivity principle [41.) This is illustrated in the schematic in Figure 6. In this paper, we shall only consider situations where this scenario holds. For examples where this breaksdown see [47, 61.

\section{Remarks:}

1. The probability (65) does not include the contribution of reservoirs. One way to do it is to consider density profiles $\rho(x)$ which are fixed at the boundary, i.e. $\rho(0, t)=\rho_{a}$ and $\rho(1, t)=\rho_{b}$. This is justified due to the strong coupling with the reservoirs, so that fluctuations of density at the boundary relax to the reservoir density in a time scale much faster than the hydrodynamic time scale.

2. The formula 67 means

$$
\chi(\kappa)=\lim _{T \rightarrow \infty} \frac{1}{T} \max _{\rho, j} S_{T}^{(\kappa, \alpha)}[\rho, j]
$$

which leads to the well-known result [35, 41]

$$
\chi(\kappa)=\max _{q}\{\kappa q-\Phi(q)\} ; \quad \Phi(q)=\min _{\bar{\rho}_{\mathrm{qs}}} \int_{0}^{1} d x \frac{\left(q+D\left(\bar{\rho}_{\mathrm{qs}}\right) \partial_{x} \bar{\rho}_{\mathrm{qs}}(x)\right)^{2}}{2 \sigma\left(\bar{\rho}_{\mathrm{qs}}\right)}
$$

Their solution [35, 41] for the independent particles and for the symmetric simple exclusion process are in agreement with 33 and 56 . 


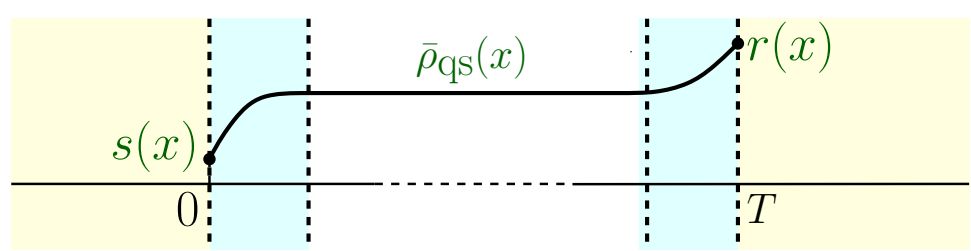

Fig. 6 A schematic of optimal evolution of density for the variatonal problem (67), where at the intermediate time the density is time independent $\bar{\rho}_{\mathrm{qs}}(x)$.

\subsection{Hamilton-Jacobi equation}

In (67), the deviation of the optimal path from $\bar{\rho}_{\mathrm{qS}}(x)$ near $t=0$ and $t=T$ (see Figure 6 are important and they contribute to $\psi_{\text {right }}^{(\kappa, \alpha)}$ and $\psi_{\text {left }}^{(\kappa, \alpha)}$. Here, we show how this variational formula (67) leads to a pair of Hamilton-Jacobi equations for $\psi_{\text {right }}^{(\kappa, \alpha)}$ and $\psi_{\text {left }}^{(\kappa, \alpha)}$.

We start by deriving the equation for $\psi_{\text {left }}^{(\kappa, \alpha)}$. For this, we use

$$
G_{T}^{(\kappa, \alpha)}[r(x), s(x)]=\int \mathcal{D}[\rho] G_{T-t}^{(\kappa, \alpha)}[r(x), \rho(x)] G_{t}^{(\kappa, \alpha)}[\rho(x), s(x)]
$$

for $0<t<T$, which can be seen from the definition (63). We consider infinitesimal $t>0$ but large $T$, such that $T-t$ is large. This means, we can use (64) for $G_{T-t}^{(\kappa, \alpha)}[r(x), \rho(x)]$. On the other hand, using the Action formulation 66 we write, for an infinitesimal $t$,

$$
G_{t}^{(\kappa, \alpha)}[\rho(x), s(x)] \sim \exp \left[t L \int_{0}^{1} d x\left\{\kappa \alpha(x) j(x)-\frac{\left(j(x)+D(s) \partial_{x} s(x)\right)^{2}}{2 \sigma(s)}\right\}\right]
$$

where $\rho(x) \simeq s(x)-t \partial_{x} j(x)$. Using this in 70 and a saddle point analysis for large $L$ we get

$$
\begin{aligned}
\psi_{\text {left }}^{(\kappa, \alpha)}[s(x)] \simeq t \chi(\kappa)- & \max _{j(x)}\left\{-\psi_{\text {left }}^{(\kappa, \alpha)}\left[s(x)-t \partial_{x} j(x)\right]\right. \\
& \left.+t \int_{0}^{1} d x\left(\kappa \alpha(x) j(x)-\frac{\left(j(x)+D(s) \partial_{x} s(x)\right)^{2}}{2 \sigma(s)}\right)\right\}
\end{aligned}
$$

Expanding $\psi_{\text {left }}^{(\kappa, \alpha)}\left[s(x)-t \partial_{x} j(x)\right]$ in a Taylor series up to linear order in $t$, and then using an integration by parts, we get

$$
\begin{aligned}
\chi(\kappa) \simeq & \max _{j(x)}\left\{\frac{\delta \psi_{\text {left }}^{(\kappa, \alpha)}}{\delta s(1)} j(1)-\frac{\delta \psi_{\mathrm{left}}^{(\kappa, \alpha)}}{\delta s(0)} j(0)\right. \\
& \left.+\int_{0}^{1} d x\left[\left(\kappa \alpha(x)-\partial_{x} \frac{\delta \psi_{\text {left }}^{(\kappa, \alpha)}}{\delta s(x)}\right) j(x)-\frac{\left(j(x)+D(s) \partial_{x} s(x)\right)^{2}}{2 \sigma(s)}\right]\right\}
\end{aligned}
$$

For density profiles which are fixed at the boundary (see the remark 1 in Section 6.1 , one can see from 28, 35, 57, that

$$
\frac{\delta \psi_{\text {left }}^{(\kappa, \alpha)}}{\delta s(x)}=0 \quad \text { at } x=0 \text { and at } x=1,
$$


for the two systems we study in this paper. Similar conditions occured already in earlier works [33, 60. Using this in (71) and optimizing over $j(x)$ leads to

$$
\int_{0}^{1} d x\left[\frac{\sigma(s)}{2}\left(\partial_{x} \frac{\delta \psi_{\text {left }}^{(\kappa, \alpha)}}{\delta s(x)}-\kappa \alpha(x)+\frac{D(s) s^{\prime}(x)}{\sigma(s)}\right)^{2}-\frac{\left(D(s) s^{\prime}(x)\right)^{2}}{2 \sigma(s)}\right]=\chi(\kappa)
$$

where we denote $s^{\prime}(x) \equiv \partial_{x} s(x)$.

A similar analysis (by considering small decrement around the time $T$ ) leads to an analogous equation for $\psi_{\text {right }}^{(\kappa, \alpha)}$.

$$
\int_{0}^{1} d x\left[\frac{\sigma(r)}{2}\left(\partial_{x} \frac{\delta \psi_{\text {right }}^{(\kappa, \alpha)}}{\delta r(x)}+\kappa \alpha(x)-\frac{D(r) r^{\prime}(x)}{\sigma(r)}\right)^{2}-\frac{\left(D(r) r^{\prime}(x)\right)^{2}}{2 \sigma(r)}\right]=\chi(\kappa)
$$

These two are the Hamilton-Jacobi equations associated to the variational problem (67).

\subsection{Optimal path}

In (71) the optimal current

$$
j_{\mathrm{opt}}(x)=-D(s) \partial_{x} s(x)+\sigma(s)\left(\kappa \alpha(x)-\partial_{x} \frac{\delta \psi_{\mathrm{left}}^{(\kappa, \alpha)}}{\delta s(x)}\right)
$$

This means that the optimal path $\rho_{\text {opt }}(x, t)$ near $t=0$ follows

$$
\partial_{t} \rho_{\mathrm{opt}}=\partial_{x}\left\{D\left(\rho_{\mathrm{opt}}\right) \partial_{x} \rho_{\mathrm{opt}}-\sigma\left(\rho_{\mathrm{opt}}\right)\left(\kappa \alpha(x)-\partial_{x} \frac{\delta \psi_{\text {left }}^{(\kappa, \alpha)}}{\delta \rho_{\mathrm{opt}}}\right)\right\}
$$

It is straightforward to extend the argument for $t \geq 0$ but $T-t \gg 1$ (region II of Figure 2 and show that the dynamics is the same.

A similar analysis in the derivation of $73 \mathrm{~b}$ shows that the optimal path $\rho_{\text {opt }}(x, t)$ in region IV of Figure 2 is described by

$$
\partial_{t} \rho_{\mathrm{opt}}=\partial_{x}\left\{D\left(\rho_{\mathrm{opt}}\right) \partial_{x} \rho_{\mathrm{opt}}-\sigma\left(\rho_{\mathrm{opt}}\right)\left(\kappa \alpha(x)+\partial_{x} \frac{\delta \psi_{\text {right }}^{(\kappa, \alpha)}}{\delta \rho_{\mathrm{opt}}}\right)\right\}
$$

In the quasi-stationary state $(76)$ also describes the optimal path leading to a fluctuation and (75) describes the optimal path of relaxation (see illustration in Figure 3. 
6.4 Fixed point of the dynamics

Using 73 we show in Appendix $\mathrm{D}$ that along the optimal path 75 ,

$$
\frac{d}{d t} \psi_{\mathrm{qs}}^{(\kappa)}\left[\rho_{\mathrm{opt}}(x, t)\right]=-\int_{0}^{1} d x \frac{\sigma\left(\rho_{\mathrm{opt}}(x, t)\right)}{2}\left(\partial_{x} \frac{\delta \psi_{\mathrm{qs}}^{(\kappa)}\left[\rho_{\mathrm{opt}}(x, t)\right]}{\delta \rho_{\mathrm{opt}}(x, t)}\right)^{2}
$$

with $\psi_{\mathrm{qs}}^{(\kappa)}$ given in $27 \mathrm{c}$. Since $\sigma(\rho)$ is positive, this means $\frac{d}{d t} \psi_{\mathrm{qs}}^{(\kappa)}\left[\rho_{\mathrm{opt}}(x, t)\right]=0$ if and only if $\partial_{x} \frac{\delta \psi_{\mathrm{qs}}^{(\kappa)}\left[\rho_{\mathrm{opt}}(x, t)\right]}{\delta \rho_{\mathrm{opt}}(x, t)}=0$. (The case $\kappa=0$ has been discussed earlier in 60.)

The examples we consider here have a unique quasi-stationary density $\bar{\rho}_{\mathrm{qs}}$, where $\frac{\delta \psi_{\mathrm{qs}}^{(\kappa)}}{\delta \bar{\rho}_{\mathrm{qs}}(x)}=0$. Then 77 implies that $\bar{\rho}_{\mathrm{qs}}$ is an attractive fixed point of 75 (see Figure 3).

For $\bar{\rho}_{\mathrm{qs}}$ the optimal current (74) is $j_{\mathrm{opt}}=\chi^{\prime}(\kappa)$, which can be seen from $(69)$. Then, we get

$$
-D\left(\bar{\rho}_{\mathrm{qs}}\right) \partial_{x} \bar{\rho}_{\mathrm{qs}}+\sigma\left(\bar{\rho}_{\mathrm{qs}}\right)\left(\kappa \alpha(x)-\partial_{x} \frac{\delta \psi_{\text {left }}^{(\kappa, \alpha)}}{\delta \bar{\rho}_{\mathrm{qs}}(x)}\right)=\chi^{\prime}(\kappa)
$$

which leads to

$$
\partial_{x} \frac{\delta \psi_{\text {left }}^{(\kappa, \alpha)}}{\delta \bar{\rho}_{\mathrm{qs}}(x)}=\kappa \alpha(x)-\frac{\chi^{\prime}(\kappa)+D\left(\bar{\rho}_{\mathrm{qs}}\right) \partial_{x} \bar{\rho}_{\mathrm{qs}}(x)}{\sigma\left(\bar{\rho}_{\mathrm{qs}}\right)}
$$

A similar calculation for 776 lead to

$$
\partial_{x} \frac{\delta \psi_{\mathrm{right}}^{(\kappa, \alpha)}}{\delta \bar{\rho}_{\mathrm{qs}}(x)}=-\kappa \alpha(x)+\frac{\chi^{\prime}(\kappa)+D\left(\bar{\rho}_{\mathrm{qs}}\right) \partial_{x} \bar{\rho}_{\mathrm{qs}}(x)}{\sigma\left(\bar{\rho}_{\mathrm{qs}}\right)}
$$

These give conditions for the solution of $(73)$. It is well-known 60, 62, that, there are multiple solutions of a Hamilton-Jacobi equation. For the two examples studied in this work, the relevant solution of 73 follows the boundary condition 78) and (see 72)

$$
\frac{\delta \psi_{\text {left }}^{(\kappa, \alpha)}}{\delta \rho(x)}=0 \quad \text { and } \quad \frac{\delta \psi_{\text {right }}^{(\kappa, \alpha)}}{\delta \rho(x)}=0 \quad \text { at } x=0 \text { and } x=1 .
$$

\subsection{Conditioned stochastic dynamics}

In Section 5 we have shown using a microscopic analysis that, in the quasistationary state, conditioned dynamics is given by a fluctuating hydrodynamics equation 62 . Here, we give a derivation using the macroscopic approach.

In the quasi-stationary state, if $\mathcal{P}\left[\rho, j \mid \rho_{i}\right]$ is the probability of a path $\{\rho(x, t), j(x, t)\}$ in a time window $\left[t_{i}, t_{f}\right]$ (for $1 \ll t_{i}<t_{f}$ and $1 \ll T-t_{f}$ ) given an initial density $\rho\left(x, t_{i}\right)=\rho_{i}(x)$, then using 63 and $66 \mathrm{~b}$ one can write

$$
\mathcal{P}\left[\rho, j \mid \rho_{i}\right]=\frac{G_{T-t_{f}}^{(\kappa, \alpha)}\left[\rho_{T}, \rho_{f}\right] e^{L S_{t_{f}-t_{i}}^{(\kappa, \alpha)}}}{G_{T-t_{i}}^{(\kappa, \alpha)}\left[\rho_{T}, \rho_{i}\right]}
$$


where we denote $\rho\left(x, t_{f}\right)=\rho_{f}(x)$ and $\rho(x, T)=\rho_{T}(x)$. Then, for large $L$, using 26) and 64 we get

$$
\mathcal{P}\left[\rho, j \mid \rho_{i}\right] \sim e^{-L\left(t_{f}-t_{i}\right) \chi(\kappa)+L \psi_{\text {left }}^{(\kappa, \alpha)}\left[\rho_{i}\right]-L \psi_{\text {left }}^{(\kappa, \alpha)}\left[\rho_{f}\right]+L S_{t_{f}-t_{i}}^{(\kappa, \alpha)}[\rho, j]}
$$

This expression can be simplified by using

$$
\begin{aligned}
\psi_{\mathrm{left}}^{(\kappa, \alpha)}\left[\rho_{f}\right]-\psi_{\mathrm{left}}^{(\kappa, \alpha)}\left[\rho_{i}\right] & =\int_{0}^{1} d x \int_{t_{i}}^{t_{f}} d t \partial_{t} \rho \frac{\delta \psi_{\text {left }}^{(\kappa, \alpha)}[\rho]}{\delta \rho(x, t)} \\
& =\int_{0}^{1} d x \int_{t_{i}}^{t_{f}} d t j(x, t) \partial_{x}\left(\frac{\delta \psi_{\text {left }}^{(\kappa, \alpha)}[\rho]}{\delta \rho(x, t)}\right)
\end{aligned}
$$

where the last equality is obtained by using $\partial_{t} \rho=-\partial_{x} j$, integration by parts, and the boundary condition 79 . In addition, we use $73 a$ to write

$\left(t_{f}-t_{i}\right) \chi(\kappa)=\int_{0}^{1} d x \int_{t_{i}}^{t_{f}} d t\left[\frac{\sigma(\rho)}{2}\left(\partial_{x} \frac{\delta \psi_{\mathrm{left}}^{(\kappa, \alpha)}}{\delta \rho(x, t)}-\kappa \alpha(x)+\frac{D(\rho) \partial_{x} \rho(x, t)}{\sigma(\rho)}\right)^{2}-\frac{\left(D(\rho) \partial_{x} \rho(x)\right)^{2}}{2 \sigma(\rho)}\right]$

Using the above two results in 80 and following a simple algebra we get

$$
\mathcal{P}\left[\rho, j \mid \rho_{i}\right] \sim e^{L \widehat{S}[\rho, j]}
$$

with the Action

$$
\widehat{S}[\rho, j]=-\int_{t_{i}}^{t_{f}} d t \int_{0}^{1} d x \frac{\left\{j(x, t)+D(\rho) \partial_{x} \rho(x, t)+\sigma(\rho)\left(\partial_{x} \frac{\delta \psi_{\text {left }}^{(\kappa, \alpha)}}{\delta \rho(x, t)}-\kappa \alpha(x)\right)\right\}^{2}}{2 \sigma(\rho)}
$$

Comparing with 65 one can clearly see that the conditioned dynamics in the quasi-stationary state is given by a fluctuating hydrodynamics equation $\partial_{t} \rho(x, t)=$ $-\partial_{x} j(x, t)$ with $j(x, t)$ in $62 \mathrm{a}$. This describes, how a spontaneous fluctuation relaxes in the quasi-stationary state.

On the other hand, $62 \mathrm{~b}$ shows the path leading to a fluctuation. This is given by a time reversal of (62a), which can be constructed (for example see eq. 2.15 of 60]) by using that $P_{\mathrm{qs}}^{(\overline{\kappa, \alpha})}[\rho]$ is the steady state of $62 \mathrm{a}$. This gives a fluctuating hydrodynamics equation $\partial_{t} \rho(x, t)=-\partial_{x}\left\{j(x, t)+\sigma(\rho) \partial_{x} \frac{\delta \psi_{\mathrm{qs}}^{(\kappa)}}{\delta \rho(x, t)}\right\}$ with the $j(x, t)$ in 62a). Then, using 27c one gets $62 \mathrm{~b}$.

\section{Solution in specific examples}

Here, we show how to check that the results for $\psi_{\text {left }}^{(\kappa, \alpha)}$ and $\psi_{\text {right }}^{(\kappa, \alpha)}$ derived in Section 3 and Section 4 using a microscopic analysis, are indeed solution of the Hamilton-Jacobi equations 73. 
7.1 Independent particles

In this case, using $(28 \mathrm{~b})$ and $(33)$ in $73 a$ we get

$$
\int_{0}^{1} d x\left[\rho(x)\left(\partial_{x} \frac{\delta V_{\mathrm{left}}^{(\kappa)}}{\delta \rho(x)}\right)^{2}+\rho^{\prime}(x)\left(\partial_{x} \frac{\delta V_{\mathrm{left}}^{(\kappa)}}{\delta \rho(x)}\right)\right]=\rho_{a}\left(e^{\kappa}-1\right)+\rho_{b}\left(e^{-\kappa}-1\right)
$$

Expression $35 \mathrm{a}$ for $V_{\text {left }}^{(\kappa)}$ gives

$$
\partial_{x} \frac{\delta V_{\text {left }}^{(\kappa)}}{\delta \rho(x)}=\frac{\left(e^{-\kappa}-1\right)}{b(x)} \quad \text { and } \quad\left(\partial_{x} \frac{\delta V_{\text {left }}^{(\kappa)}}{\delta \rho(x)}\right)^{2}=\left(e^{-\kappa}-1\right)\left(\frac{1}{b(x)}\right)^{\prime}
$$

With this, the left hand side of 82 becomes

$$
\begin{aligned}
\left(e^{-\kappa}-1\right) \int_{0}^{1} d x\left[\rho(x)\left(\frac{1}{b(x)}\right)^{\prime}+\frac{\rho^{\prime}(x)}{b(x)}\right] & =\left(e^{-\kappa}-1\right) \int_{0}^{1} d x\left(\frac{\rho(x)}{b(x)}\right)^{\prime} \\
& =\left(e^{-\kappa}-1\right)\left(\frac{\rho(1)}{b(1)}-\frac{\rho(0)}{b(0)}\right)
\end{aligned}
$$

From the boundary condition $\rho(0)=\rho_{a}, \rho(1)=\rho_{b}$, and using $35 \mathrm{~b}$ we see that the above expression agrees with the right hand side of $(82)$.

Moreover, one can check that the solution 28b 35a is consistent with the boundary condition 78,79 .

A similar calculation shows that $\psi_{\text {right }}^{(\kappa)}$ in $\left.28 \mathrm{a} 34 \mathrm{a}\right)$ is a solution of $(73 \mathrm{~b})$ with the boundary condition 78,79 .

\subsection{Symmetric simple exclusion process}

In this case, our solution for $\psi_{\text {left }}^{(\kappa, \alpha)}$ and $\psi_{\text {right }}^{(\kappa, \alpha)}$ in $28,57,58$ are for small density. We have explicitly verified, up to the second order in density, that these are solutions of the Hamilton-Jacobi equations (73) and they satisfy the boundary condition 78 .790 . The analysis is similar to that of the independent particles in Section 7.1 In fact, at the leading order in density, they are identical.

\section{Summary}

In the present work we have tried to determine the probability of the density (4) in a diffusive many-particle system conditioned on the time-integrated current (3) for large $T$. This is a generalization to extended systems of earlier works on conditioned stochastic processes [8, 16, 22, 28, 31]. We mostly worked with the canonical ensemble where dynamics is weighted by the current (3). However, the equivalence of ensembles allows to make predictions for the conditioned process (see Section 2). We give explicit results for a system of independent particles (Section 3) and the symmetric simple exclusion process (Section 4).

In the hydrodynamic limit, the conditioned probability of the density $\rho(x)$ is characterized by the large deviation function $\psi_{t}^{(\kappa, \alpha)}[\rho]$ in 26 . For the two systems 
considered in this paper, we have calculated $\psi_{t}^{(\kappa, \alpha)}[\rho]$ at three different times of the evolution, namely, at $t=0$, at $t=T$, and in the quasi-stationary regime. These are, in general, related by (see (27))

$$
\psi_{0}^{(\kappa, \alpha)}[\rho]+\psi_{T}^{(\kappa, \alpha)}[\rho]=\psi_{\mathrm{qs}}^{(\kappa, \alpha)}[\rho]+\mathcal{F}[\rho]
$$

with $\mathcal{F}$ defined in 2 .

In the second half of the paper, we used a macroscopic approach, where $\psi_{t}^{(\kappa, \alpha)}$ is expressed (see (27)) in terms of $\psi_{\text {left }}^{(\kappa, \alpha)}$ and $\psi_{\text {right }}^{(\kappa, \alpha)}$, which are solutions of a pair of Hamilton-Jacobi equations 73 . These solutions also act as the potential for an additional field that drives the conditioned process (see $(62)$ and Section 6.5). Using this macroscopic approach we verified the microscopic results for the two specific examples (see Section 7).

The macroscopic formulation is expected to work for a wide class of diffusive systems where the microscopic details enter in the two functions $D(\rho)$ and $\sigma(\rho)$ in (1). This is in the spirit of the macroscopic fluctuation theory [33]. It would be interesting find more examples for explicit solutions of $\psi_{\text {left }}^{(\kappa, \alpha)}$ and $\psi_{\text {right }}^{(\kappa, \alpha)}$ using both the microscopic and the macroscopic approach. For systems on a ring, where the unconditioned state is in equilibrium, the calculation may be simpler. However, due to the periodic boundary condition, the optimal profile in the quasi-stationary state could become time dependent (see [47, 61, 63).

In the unconditioned case $(\kappa=0)$, the spatial correlations of density follow simple differential equations [38, 64, whose solutions can be formally expressed in terms of a Green's function. It would be interesting to see if there are similar equations for the conditioned case, especially in the quasi-stationary state.

Acknowledgements We thank T. Bodineau for his useful comments related to $27 \mathrm{c}$ and 83.

\section{A Tilted matrix for the symmetric simple exclusion process}

Here, we explicitly write the eigenvalue equation for the tilted Matrix in a symmetric simple exclusion process of arbitrary length $L$. Our results are for the case $\lambda_{0}=1$ and $\lambda_{i}=0$ for $i \geq 1$. We use the representation $\sqrt{39}$ for the eigenvectors with a normalization such that the component of both right and left eigenvectors for the empty configuration is 1 . We write the eigenvalue equation up to the two particle sector.

For the right eigenvector, defining $\mathcal{R}^{(\kappa)}(i, j)=0$ for $i=j$, we get the following set of coupled equations.

- Empty-particle sector.

$$
\mu+\rho_{a}+\rho_{b}=e^{-\kappa}\left(1-\rho_{a}\right) \mathcal{R}^{(\kappa)}(1)+\left(1-\rho_{b}\right) \mathcal{R}^{(\kappa)}(L)
$$

- Single-particle sector.

○ For $1<i<L$,

$$
\begin{aligned}
\left(\mu+\rho_{a}+\rho_{b}\right) \mathcal{R}^{(\kappa)}(i) & -\left[\mathcal{R}^{(\kappa)}(i-1)-2 \mathcal{R}^{(\kappa)}(i)+\mathcal{R}^{(\kappa)}(i+1)\right] \\
= & e^{-\kappa}\left(1-\rho_{a}\right) \mathcal{R}^{(\kappa)}(1, i)+\left(1-\rho_{b}\right) \mathcal{R}^{(\kappa)}(i, L)
\end{aligned}
$$

○ For $i=1$,

$$
\begin{aligned}
\left(\mu+\rho_{a}+\rho_{b}\right) \mathcal{R}^{(\kappa)}(1)- & {\left[2 \rho_{a} \mathcal{R}^{(\kappa)}(1)-2 \mathcal{R}^{(\kappa)}(1)+\mathcal{R}^{(\kappa)}(2)\right] } \\
= & e^{\kappa} \rho_{a}+\left(1-\rho_{b}\right) \mathcal{R}^{(\kappa)}(1, L)
\end{aligned}
$$


- For $i=L$

$$
\begin{gathered}
\left(\mu+\rho_{a}+\rho_{b}\right) \mathcal{R}^{(\kappa)}(L)-\left[\mathcal{R}^{(\kappa)}(L-1)-2 \mathcal{R}^{(\kappa)}(L)+2 \rho_{b} \mathcal{R}^{(\kappa)}(L)\right] \\
=e^{-\kappa}\left(1-\rho_{a}\right) \mathcal{R}^{(\kappa)}(1, L)+\rho_{b}
\end{gathered}
$$

- Two-particle sector.

- For $1<i<j<L$,

$$
\begin{gathered}
\left(\mu+\rho_{a}+\rho_{b}\right) \mathcal{R}^{(\kappa)}(i, j)-\left[\mathcal{R}^{(\kappa)}(i-1, j)+\mathcal{R}^{(\kappa)}(i, j-1)-4 \mathcal{R}^{(\kappa)}(i, j)\right. \\
\left.+\mathcal{R}^{(\kappa)}(i+1, j)+\mathcal{R}^{(\kappa)}(i, j+1)\right]-2 \mathcal{R}^{(\kappa)}(i, j) \delta_{i+1, j} \\
=e^{-\kappa}\left(1-\rho_{a}\right) \mathcal{R}^{(\kappa)}(1, i, j)+\left(1-\rho_{b}\right) \mathcal{R}^{(\kappa)}(i, j, L)
\end{gathered}
$$

○ For $1=i<j<L$,

$$
\begin{aligned}
\left(\mu+\rho_{a}\right. & \left.+\rho_{b}\right) \mathcal{R}^{(\kappa)}(1, j)-\left[2 \rho_{a} \mathcal{R}^{(\kappa)}(1, j)+\mathcal{R}^{(\kappa)}(1, j-1)-4 \mathcal{R}^{(\kappa)}(1, j)+\mathcal{R}^{(\kappa)}(2, j)\right. \\
& \left.+\mathcal{R}^{(\kappa)}(1, j+1)\right]-2 \mathcal{R}^{(\kappa)}(1, j) \delta_{2, j}=e^{\kappa} \rho_{a} \mathcal{R}^{(\kappa)}(j)+\left(1-\rho_{b}\right) \mathcal{R}^{(\kappa)}(1, j, L)
\end{aligned}
$$

○ For $1<i<j=L$,

$$
\begin{aligned}
\left(\mu+\rho_{a}\right. & \left.+\rho_{b}\right) \mathcal{R}^{(\kappa)}(i, L)-\left[\mathcal{R}^{(\kappa)}(i-1, L)+\mathcal{R}^{(\kappa)}(i, L-1)-4 \mathcal{R}^{(\kappa)}(i, L)+\mathcal{R}^{(\kappa)}(i+1, L)\right. \\
& \left.+2 \rho_{b} \mathcal{R}^{(\kappa)}(i, L)\right]-2 \mathcal{R}^{(\kappa)}(i, L) \delta_{i, L-1}=e^{-\kappa}\left(1-\rho_{a}\right) \mathcal{R}^{(\kappa)}(1, i, L)+\rho_{b} \mathcal{R}^{(\kappa)}(i)
\end{aligned}
$$

Similarly, for the left eigenvector, we define $\mathcal{L}^{(\kappa)}(i, j)=0$ for $i=j$, and get the following set of equations.

- Empty particle sector.

$$
\mu+\rho_{a}+\rho_{b}=e^{\kappa} \rho_{a} \mathcal{L}^{(\kappa)}(1)+\rho_{b} \mathcal{L}^{(\kappa)}(L)
$$

- Single particle sector.

○ For $1<i<L$,

$$
\begin{aligned}
\left(\mu+\rho_{a}+\rho_{b}\right) \mathcal{L}^{(\kappa)}(i) & -\left[\mathcal{L}^{(\kappa)}(i-1)-2 \mathcal{L}^{(\kappa)}(i)+\mathcal{L}^{(\kappa)}(i+1)\right] \\
& =e^{\kappa} \rho_{a} \mathcal{L}^{(\kappa)}(1, i)+\rho_{b} \mathcal{L}^{(\kappa)}(i, L)
\end{aligned}
$$

○ For $i=1$,

$$
\begin{aligned}
\left(\mu+\rho_{a}+\rho_{b}\right) \mathcal{L}^{(\kappa)}(1)- & {\left[2 \rho_{a} \mathcal{L}^{(\kappa)}(1)-2 \mathcal{L}^{(\kappa)}(1)+\mathcal{L}^{(\kappa)}(2)\right] } \\
= & e^{-\kappa}\left(1-\rho_{a}\right)+\rho_{b} \mathcal{L}^{(\kappa)}(1, L)
\end{aligned}
$$

○ For $i=L$,

$$
\begin{aligned}
\left(\mu+\rho_{a}+\rho_{b}\right) \mathcal{L}^{(\kappa)}(L) & -\left[\mathcal{L}^{(\kappa)}(L-1)-2 \mathcal{L}^{(\kappa)}(L)+2 \rho_{b} \mathcal{L}^{(\kappa)}(L)\right] \\
& =e^{\kappa} \rho_{a} \mathcal{L}^{(\kappa)}(1, L)+\left(1-\rho_{b}\right)
\end{aligned}
$$

- Two particle sector.

○ For $1<i<j<L$,

$$
\begin{gathered}
\left(\mu+\rho_{a}+\rho_{b}\right) \mathcal{L}^{(\kappa)}(i, j)-\left[\mathcal{L}^{(\kappa)}(i-1, j)+\mathcal{L}^{(\kappa)}(i, j-1)-4 \mathcal{L}^{(\kappa)}(i, j)\right. \\
\left.+\mathcal{L}^{(\kappa)}(i+1, j)+\mathcal{L}^{(\kappa)}(i, j+1)\right]-2 \mathcal{L}^{(\kappa)}(i, j) \delta_{i+1, j} \\
=e^{\kappa} \rho_{a} \mathcal{L}^{(\kappa)}(1, i, j)+\rho_{b} \mathcal{L}^{(\kappa)}(i, j, L)
\end{gathered}
$$


○ For $1=i<j<L$,

$$
\begin{aligned}
\left(\mu+\rho_{a}\right. & \left.+\rho_{b}\right) \mathcal{L}^{(\kappa)}(1, j)-\left[2 \rho_{a} \mathcal{L}^{(\kappa)}(1, j)+\mathcal{L}^{(\kappa)}(1, j-1)-4 \mathcal{L}^{(\kappa)}(1, j)+\mathcal{L}^{(\kappa)}(2, j)\right. \\
& \left.+\mathcal{L}^{(\kappa)}(1, j+1)\right]-2 \mathcal{L}^{(\kappa)}(1, j) \delta_{2, j}=e^{-\kappa}\left(1-\rho_{a}\right) \mathcal{L}^{(\kappa)}(j)+\rho_{b} \mathcal{L}^{(\kappa)}(1, j, L)
\end{aligned}
$$

○ For $1<i<j=L$,

$$
\begin{gathered}
\left(\mu+\rho_{a}+\rho_{b}\right) \mathcal{L}^{(\kappa)}(i, L)-\left[\mathcal{L}^{(\kappa)}(i-1, L)+\mathcal{L}^{(\kappa)}(i, L-1)-4 \mathcal{L}^{(\kappa)}(i, L)+\mathcal{L}^{(\kappa)}(i+1, L)\right. \\
\left.+2 \rho_{b} \mathcal{L}^{(\kappa)}(i, L)\right]-2 \mathcal{L}^{(\kappa)}(i, L) \delta_{i, L-1}=e^{\kappa} \rho_{a} \mathcal{L}^{(\kappa)}(1, i, L)+\left(1-\rho_{b}\right) \mathcal{L}^{(\kappa)}(i)
\end{gathered}
$$

It is easy to see a pattern in the equations and using this we can write the equations for an arbitrary particle sector. It is then possible to systematically solve the equations order by order using the perturbation expansion in small density as given in Section 4.2

\section{B Cumulants for the symmetric simple exclusion process}

We write the cumulants of density using a set of parameters

$$
z=e^{\kappa}, \quad h=e^{\kappa}-1, \quad s=\rho_{a} h, \quad \text { and } \quad p=1-\frac{\rho_{b}}{\rho_{a} z}
$$

which was used earlier [51. In terms of these, the cumulants in 49 have the following expression.

- At $t=0$

$$
\begin{array}{r}
\bar{\rho}(x)=\frac{\rho_{a}}{z}\left\{(1+x h)(1+x h-(p z) x)+\frac{s}{6 z}(1-x)[6+2 x h(7-z+x h(6-z+2 x h))\right. \\
\left.\left.-(p z) x(10+2 z+x h(16-2 z+7 x h))+(p z)^{2} x(2+x(5+3 x h))\right]+\mathcal{O}\left(s^{2}\right)\right\} \\
c(x, y)=-\frac{\rho_{a}^{2}}{z^{2}} x(1-y)\left\{2 h^{2}(1+x h)(1+y h)-h(p z)\left(2+3 h(x+y)+4 h^{2} x y\right)\right. \\
\left.+(p z)^{2}\left(1+h(x+y)+2 h^{2} x y\right)+\mathcal{O}(s)\right\} \quad \text { for } x \leq y .
\end{array}
$$

- In the quasi-stationary state,

$$
\begin{aligned}
\bar{\rho}(x) \equiv \bar{\rho}_{\mathrm{qS}}(x) & =\rho_{a}\{(1-p x)(1+x h) \\
& \left.-s x(1-x)\left[h+\frac{p}{3}(7-z+2 x h)-\frac{p^{2}}{3}(1+x)(2+x h)\right]+\mathcal{O}\left(s^{2}\right)\right\}, \\
c(x, y) & =-\rho_{a}^{2} x(1-y)\left\{p^{2}(1+h x)(1+h y)+h^{2}(1-p x)(1-p y)+\mathcal{O}(s)\right\} \quad \text { for } x \leq y .
\end{aligned}
$$

- at $t=T$,

$$
\begin{aligned}
\bar{\rho}(x) & =\rho_{a} z\left\{1-p x+s \frac{(1-x)}{6}\left[-6+p^{2} x(2-x)\right]+\mathcal{O}\left(s^{2}\right)\right\}, \\
c(x, y) & =-\rho_{a}^{2} z^{2} x(1-y)\left\{p^{2}+\mathcal{O}(s)\right\} \quad \text { for } x \leq y .
\end{aligned}
$$




\section{Small density expansion of $V_{\text {left }}^{(\kappa)}$}

Here, we show that the expression of $V_{\text {left }}^{(\kappa)}$ in $(57)$ agrees with $\psi_{\mathrm{qs}}^{(\kappa)}-\psi_{T}^{(\kappa)}$, up to an additive constant, as expected from $27 \mathrm{c}$. To see this, we use $55 \mathrm{a}$ and we get

$$
\begin{aligned}
\psi_{\mathrm{qs}}^{(\kappa)}[\rho]-\psi_{T}^{(\kappa)}[\rho]= & \int_{0}^{1} d x \log \frac{1-\bar{\rho}_{T}(x)}{1-\bar{\rho}_{\mathrm{qS}}(x)}+\frac{1}{2} \int_{0}^{1} d x \int_{0}^{1} d y\left\{c_{T}(x, y)-c_{\mathrm{qs}}(x, y)\right\} \\
& +\int_{0}^{1} d x \rho(x) \log \left[\frac{\bar{\rho}_{T}(x)}{1-\bar{\rho}_{T}(x)} \frac{1-\bar{\rho}_{\mathrm{qs}}(x)}{\bar{\rho}_{\mathrm{qs}}(x)}\right] \\
& -\int_{0}^{1} d x \rho(x) \int_{0}^{1} d y\left\{\frac{c_{T}(x, y)}{\bar{\rho}_{T}(x)}-\frac{c_{\mathrm{qs}}(x, y)}{\bar{\rho}_{\mathrm{qS}}(x)}\right\} \\
& -\frac{1}{2} \int_{0}^{1} d x \int_{0}^{1} d y \rho(x) \rho(y)\left\{\frac{c_{\mathrm{qS}}(x, y)}{\bar{\rho}_{\mathrm{qs}}(x) \bar{\rho}_{\mathrm{qs}}(y)}-\frac{c_{T}(x, y)}{\bar{\rho}_{T}(x) \bar{\rho}_{T}(y)}\right\}+\cdots
\end{aligned}
$$

where the subscripts $T$ and qs refer to the cumulants 49 at time $t=T$ and in the quasistationary state. (In the third line of the above equation we used that $c_{T}(x, y)$ and $c_{\mathrm{qs}}(x, y)$ are symmetric under exchange of $x$ and $y$.) The terms in the first line are constant and therefore ignored. For the rest of the terms we use

$$
\begin{aligned}
c_{\mathrm{qS}}(x, y) \simeq & \ell^{(\kappa)}(x) \ell^{(\kappa)}(y) c_{T}(x, y)+\bar{\rho}_{T}(x) \bar{\rho}_{T}(y) g_{\mathrm{left}}^{(\kappa)}(x, y) \\
\bar{\rho}_{\mathrm{qs}}(x) \simeq & \ell^{(\kappa)}(x) \bar{\rho}_{T}(x)\left[1-\ell^{(\kappa)}(x) \bar{\rho}_{T}(x)\right]+\ell^{(\kappa)}(x) \bar{\rho}_{T}(x)^{2} \\
& -\ell^{(\kappa)}(x) \int_{0}^{1} d y\left(1-\ell^{(\kappa)}(y)\right) c_{T}(x, y)+\bar{\rho}_{T}(x) \int_{0}^{1} d y \bar{\rho}_{T}(y) g_{\text {left }}^{(\kappa)}(x, y)
\end{aligned}
$$

up to the second order in $\rho_{a}$ and $\rho_{b}$, which can be seen from 51 and 52 .

Then, it is straightforward to see that, up to the second order in $\rho_{a}$ and $\rho_{b}$,

$$
\rho(x) \rho(y)\left\{\frac{c_{\mathrm{qs}}(x, y)}{\bar{\rho}_{\mathrm{qs}}(x) \bar{\rho}_{\mathrm{qs}}(y)}-\frac{c_{T}(x, y)}{\bar{\rho}_{T}(x) \bar{\rho}_{T}(y)}\right\} \simeq \rho(x) \rho(y) \frac{g_{\mathrm{left}}^{(\kappa)}(x, y)}{\ell^{(\kappa)}(x) \ell^{(\kappa)}(y)}
$$

and

$$
\log \left[\frac{\bar{\rho}_{T}(x)}{1-\bar{\rho}_{T}(x)} \frac{1-\bar{\rho}_{\mathrm{qS}}(x)}{\bar{\rho}_{\mathrm{qS}}(x)}\right]-\int_{0}^{1} d y\left\{\frac{c_{T}(x, y)}{\bar{\rho}_{T}(x)}-\frac{c_{\mathrm{qS}}(x, y)}{\bar{\rho}_{\mathrm{qS}}(x)}\right\} \simeq \log \frac{1}{\ell^{(\kappa)}(x)}
$$

This shows that the expression in 85 agrees with $V_{\text {left }}^{(\kappa)}$ in 57 , up to an additive constant.

\section{A H-theorem}

To derive 777 we write, along the optimal path 75 ,

$$
\frac{d}{d t} \psi_{\mathrm{qs}}^{(\kappa, \alpha)}=\int_{0}^{1} d x \frac{\delta \psi_{\mathrm{qs}}^{(\kappa, \alpha)}}{\delta \rho_{\mathrm{opt}}} \partial_{t} \rho_{\mathrm{opt}}
$$

Then, using 75 and an integration by parts we get

$$
\frac{d}{d t} \psi_{\mathrm{qs}}^{(\kappa, \alpha)}=-\int_{0}^{1} d x\left\{\partial_{x} \frac{\delta \psi_{\mathrm{qs}}^{(\kappa, \alpha)}}{\delta \rho_{\mathrm{opt}}}\right\}\left\{D\left(\rho_{\mathrm{opt}}\right) \partial_{x} \rho_{\mathrm{opt}}+\sigma\left(\rho_{\mathrm{opt}}\right)\left(\partial_{x} \frac{\delta \psi_{\mathrm{left}}^{(\kappa, \alpha)}}{\delta \rho_{\mathrm{opt}}}-\kappa \alpha(x)\right)\right\}
$$


where we use $\frac{\delta \psi_{\mathrm{qs}}^{(\kappa, \alpha)}}{\delta \rho_{\mathrm{opt}}(x, t)}=0$ at the boundary $x=0$ and 1 (due to $27 \mathrm{c}$ and 79 ). To simplify the expression, we use

$\int_{0}^{1} d x\left\{\partial_{x} \frac{\delta \psi_{\mathrm{qs}}^{(\kappa, \alpha)}}{\delta \rho_{\mathrm{opt}}}\right\}\left\{D\left(\rho_{\mathrm{opt}}\right) \partial_{x} \rho_{\mathrm{opt}}+\frac{\sigma\left(\rho_{\mathrm{opt}}\right)}{2}\left(\partial_{x} \frac{\delta \psi_{\mathrm{left}}^{(\kappa, \alpha)}}{\delta \rho_{\mathrm{opt}}}-\partial_{x} \frac{\delta \psi_{\mathrm{right}}^{(\kappa, \alpha)}}{\delta \rho_{\mathrm{opt}}}-2 \kappa \alpha(x)\right)\right\}=0$

which is obtained by subtracting $73 \mathrm{~b}$ from $73 \mathrm{a}$ and then using $27 \mathrm{c}$. From the above two equations we get 77 .

\section{References}

1. Jack R L and Sollich P 2010 Prog. Theo. Phys. Sup. 184304

2. Majumdar S N and Orland H 2015 J. Stat. Mech. P06039

3. Hirschberg O, Mukamel D and Schütz G M 2015 J. Stat. Mech. P11023

4. Schütz G M 2016 Duality Relations for the Periodic ASEP Conditioned on a Low Current (Cham: Springer International Publishing) p 323

5. Popkov V and Schütz G M 2011 J. Stat. Phys. 142627

6. Popkov V, Schütz G M and Simon D 2010 J. Stat. Mech. P10007

7. Touchette H 2009 Phys. Rep. 4781

8. Chetrite R and Touchette H 2015 Ann. Henri Poincaré 162005

9. Maes C 1999 J. Stat. Phys. 95367

10. Mey A S J S, Geissler P L and Garrahan J P 2014 Phys. Rev. E 89032109

11. Delarue M, Koehl P and Orland H 2017 J. Chem. Phys. 147152703

12. Dykman M I, Mori E, Ross J and Hunt P M 1994 J. Chem. Phys. 1005735

13. Horowitz J M and Kulkarni R V 2017 Physical Biology 14 03LT01

14. Lauri J and Bouchet F 2015 N. J. Phys 17015009

15. Garrahan J P, Jack R L, Lecomte V, Pitard E, van Duijvendijk K and van Wijland F 2007 Phys. Rev. Lett. 98195702

16. Garrahan J P, Jack R L, Lecomte V, Pitard E, van Duijvendijk K and van Wijland F 2009 J. Phys. A 42075007

17. Dorlas T C and Wedagedera J R 2001 Int. J. Mod. Phys. B 151

18. Ray U, Chan G K and Limmer D T 2018 Phys. Rev. Lett. 120210602

19. Giardina C, Kurchan J, Lecomte V and Tailleur J 2011 J. Stat. Phys. 145787

20. Tailleur J and Lecomte V 2009 AIP Conference Proceedings 1091212

21. Lebowitz J L and Spohn H 1999 J. Stat. Phys. 95333

22. Touchette H 2018 Physica A 5045 lecture Notes of the 14th International Summer School on Fundamental Problems in Statistical Physics

23. Maes C and Netocný K 2008 EPL 8230003

24. Maes C, Netocnný K and Wynants B 2008 Physica A 3872675

25. Mehl J, Speck T and Seifert U 2008 Phys. Rev. E 78011123

26. Speck T, Engel A and Seifert U 2012 J. Stat. Mech. P12001

27. Agranov T, Krapivsky P L and Meerson B 2019 arXiv 1901.00153

28. Derrida B and Sadhu T 2018 arXiv $\mathbf{1 8 0 7 . 0 6 5 4 3}$

29. Jack R L and Sollich P 2015 Euro. Phys. J. Special Topics 2242351

30. Chetrite R and Touchette H 2013 Phys. Rev. Lett. 111120601

31. Lecomte V, Appert-Rolland C and van Wijland F 2007 J. Stat. Phys. 12751

32. Evans R M L 2004 Phys. Rev. Lett. 92150601

33. Bertini L, De Sole A, Gabrielli D, Jona-Lasinio G and Landim C 2015 Rev. Mod. Phys. 87593

34. Bertini L, De Sole A, Gabrielli D, Jona-Lasinio G and Landim C 2009 J. Stat. Phys. 135 857

35. Derrida B 2007 J. Stat. Mech. P07023

36. Eyink G, Lebowitz J L and Spohn H 1990 Comm. Math. Phys. 132253

37. Spohn H 1991 Large Scale Dynamics of Interacting Particles (New York: Springer-Verlag)

38. Sadhu T and Derrida B 2016 J. Stat. Mech. 113202

39. Bertini L, De Sole A, Gabrielli D, Jona-Lasinio G and Landim C 2001 Phys. Rev. Lett. 87040601 
40. Tailleur J, Kurchan J and Lecomte V 2008 J. Phys. A 41505001

41. Bodineau T and Derrida B 2004 Phys. Rev. Lett. 92180601

42. Bodineau T and Derrida B 2005 Phys. Rev. E 72066110

43. Derrida B, Lebowitz J L and Speer E R 2001 Phys. Rev. Lett. 87150601

44. Hurtado P I and Garrido P L 2010 Phys. Rev. E 81041102

45. Hurtado P I, Espigares C P, del Pozo J J and Garrido P L 2014 J. Stat. Phys. 154214

46. Bertini L, Sole A D, Gabrielli D, Jona-Lasinio G and Landim C 2006 J. Stat. Phys. 123 237

47. Bertini L, De Sole A, Gabrielli D, Jona-Lasinio G and Landim C 2007 Theory of Probability E) Its Applications $\mathbf{5 1} 2$

48. Bertini L, De Sole A, Gabrielli D, Jona-Lasinio G and Landim C 2005 Phys. Rev. Lett. 94030601

49. Chetrite R and Touchette H 2015 J. Stat. Mech. P12001

50. Evans R M L 2004 J. Phys. A 38293

51. Derrida B, Douçot B and Roche P E 2004 J. Stat. Phys. 115717

52. Kipnis C and Landim C 1999 Scaling Limits of Interacting Particle Systems (Berlin: Springer-Verlag)

53. Bodineau T Private communication

54. van Kampen N G 2007 Stochastic Processes in Physics and Chemistry 3rd ed (Amsterdam: Elsevier)

55. Lazarescu A 2015 J. Phys. A 48503001

56. Vanicat M 2018 An integrabilist approach of out-of-equilibrium statistical physics models Ph.D. thesis Université Grenoble Alpes HAL Id: tel-01688587 https://tel.archivesouvertes.fr/tel-01688587

57. Derrida B, Douçot B and Roche P E 2004 J. Stat. Phys. 115717

58. Stroock D W 2014 An Introduction to Markov Processes 2nd ed (Graduate Texts in Mathematics vol 230) (Heidelberg: Springer)

59. Bodineau T, Derrida B and Lebowitz J 2010 J. Stat. Phys. 140648

60. Bertini L, De Sole A, Gabrielli D and Landim C 2002 J. Stat. Phys. 107635

61. Bodineau T and Derrida B 2007 C. R. Physique 8540

62. Goldstein H, Poole C and Safko J 2000 Classical Mechanics 3rd ed (New York: Addison Wesley)

63. Tizón-Escamilla N, Lecomte V and Bertin E 2019 J. Stat. Mech. 2019013201

64. Bertini L, De Sole A, Gabrielli D, Jona-Lasinio G and Landim C 2007 arXiv 0705.2996 